\title{
PHOTONICS Research
}

\section{Hybrid photonic-plasmonic cavities based on the nanoparticle-on-a-mirror configuration}

\author{
Angela I. Barreda, ${ }^{1,2,3}$ (i) Mario Zapata-Herrera, ${ }^{4,5}$ Isabelle M. Palstra, ${ }^{6,7}$ Laura Mercadé, ${ }^{3}$ \\ Javier Aizpurua, ${ }^{4,5}$ A. Femius Koenderink, ${ }^{6,7}$ (i) and Alejandro Martínez ${ }^{3, *}$ (i) \\ ${ }^{1}$ Institute of Solid State Physics, Friedrich Schiller University Jena, 07743 Jena, Germany \\ ${ }^{2}$ Institute of Applied Physics, Abbe Center of Photonics, Friedrich Schiller University Jena, 07745 Jena, Germany \\ ${ }^{3}$ Nanophotonics Technology Center, Universitat Politècnica de València, Camino de Vera s/n, 46022 Valencia, Spain \\ ${ }^{4}$ Materials Physics Center CSIC-UPVIEHU, 20018 Donostia-San Sebastian, Spain \\ ${ }^{5}$ Donostia International Physics Center DIPC, 20018 Donostia-San Sebastian, Spain \\ ${ }^{6}$ Center for Nanophotonics, AMOLF, 1098 XG Amsterdam, The Netherlands \\ ${ }^{7}$ Van der Waals-Zeeman Institute, Institute of Physics, University of Amsterdam, 1090 GL Amsterdam, The Netherlands \\ *Corresponding author: amartinez@ntc.upv.es
}

Received 9 June 2021; revised 15 September 2021; accepted 16 September 2021; posted 21 September 2021 (Doc. ID 433761); published 15 November 2021

\begin{abstract}
Hybrid photonic-plasmonic cavities have emerged as a new platform to increase light-matter interaction capable to enhance the Purcell factor in a singular way not attainable with either photonic or plasmonic cavities separately. In the hybrid cavities proposed so far, the plasmonic element is usually a metallic bow-tie antenna, so the plasmonic gap-defined by lithography-is limited to minimum values of several nanometers. Nanoparticleon-a-mirror (NPoM) cavities are far superior to achieve the smallest possible mode volumes, as plasmonic gaps smaller than $1 \mathrm{~nm}$ can be created. Here, we design a hybrid cavity that combines an NPoM plasmonic cavity and a dielectric-nanobeam photonic crystal cavity operating at transverse-magnetic polarization. The metallic nanoparticle can be placed very close $(<1 \mathrm{~nm})$ to the upper surface of the dielectric cavity, which acts as a low-reflectivity mirror. We demonstrate through numerical calculations of the local density of states that this hybrid plasmonicphotonic cavity exhibits quality factors $Q$ above $10^{3}$ and normalized mode volumes $V$ down to $10^{-3}$, thus resulting in high Purcell factors $\left(F_{P} \approx 10^{5}\right)$, while being experimentally feasible with current technology. Our results suggest that hybrid cavities with sub-nanometer gaps should open new avenues for boosting light-matter interaction in nanophotonic systems. () 2021 Chinese Laser Press
\end{abstract}

https://doi.org/10.1364/PRJ.433761

\section{INTRODUCTION}

Optical cavities are structures tailored to localize light in small volumes during long residence times. To quantify light-matter interaction in optical cavities, a key metric is the Purcell factor $\left(F_{P}\right)[1-3]$, which is proportional to the ratio between the quality factor $Q$ (or $Q$ factor) and the effective volume of the cavity mode, quantifying the local density of optical states (LDOS) at resonance $[4,5]$

$$
F_{P}=\frac{3}{4 \pi^{2}} \frac{Q}{V},
$$

where $V$ is mode volume normalized by the wavelength $(\lambda)$ over the local refractive index $(n)$ cubed $(\lambda / n)^{3}$, and the $Q$ factor measures the lifetime of a photon in the cavity in units of optical cycles. Dielectric cavities made of transparent materials enable very large $Q$ values [6] since photons can remain for a long time inside the cavity without dissipation [7]. However, the mode volume, which accounts for the spatial extension of the electromagnetic field inside a cavity, has a diffractionlimited floor $V \approx 1$. Thus, reaching subwavelength confinement $(V<1)$ requires the use of metallic cavities with nanometer $(\mathrm{nm})$-scale plasmonic gaps $[8,9]$. This allows us to overcome the diffraction limit at the expense of having low $Q$ factors (around 10) as a result of the large absorption and scattering losses provided by the metal. Basically, this constrains optimization of the Purcell factor: when increasing cavity storage times in a dielectric cavity, the spatial confinement cannot be extreme. Conversely, extreme subwavelength spatial confinement in nm-scale plasmonic gaps comes at the cost of very short photon lifetimes, thus reducing the $Q$ factor $[10,11]$.

In the last few years, hybrid plasmonic-photonic cavities $[10,12-21]$ have emerged as a promising way of mixing both types of confinement approaches, taking advantage of the idea of placing a plasmonic nanoantenna in a large field confinement 
region of a dielectric cavity where both modes, plasmonic and photonic, can hybridize. This results in new features not attainable by either plasmonic or photonic cavities when operating individually. Interestingly, such cavities enable engineering the LDOS by properly tailoring the coupling between the plasmonic and photonic modes so that the $Q$ factor and the normalized mode volume $V$ can be tuned [16]. This also allows us to harness the value of the Purcell factor so that it can be suitably chosen for different applications in classical and quantum optics. For the latter, previous works have mainly focused on single-photon sources, low-threshold lasers, strong coupling with quantum emitters, or sensing and vibrational spectroscopy [16,22-25].

Among the different possible implementations of such hybrids, the integration of bow-tie nanoantennas as canonical plasmonic structures on dielectric photonic crystal cavities has a set of advantages [16], such as the low value of the mode volume of the photonic cavity and the possibility of fabrication using conventional lithography methods. Noticeably, such hybrid cavities have not yet witnessed an experimental demonstration, although several experiments have confirmed the integration of bow-tie nanoantennas in dielectric waveguides [26-31]. A main limitation of this hybrid plasmonic-photonic cavity approach is that the bow-tie gap is defined by lithography, which limits its minimum attainable value. Whilst reaching gap widths below $10 \mathrm{~nm}$ is attainable [32-34], repeatability is very poor: given a same nominal value, the obtained gap width is extremely dependent on the local conditions and can take different values - or get closed-for different bow-tie nanoantennas, even when fabricated in same lithography step $[27,34]$. In general, reaching $\mathrm{nm}$ and sub-nm plasmonic gaps in a controllable fashion becomes extremely complex and hardly reconcilable with the multistep lithography challenge of integration with photonic cavities.

A much more appealing approach to reach $\mathrm{nm}$ - and sub-nm-scale plasmonic gaps in a repeatable way is by vertical deposition. Within this paradigm, nanoparticle-on-a-mirror $(\mathrm{NPoM})$ plasmonic cavities have demonstrated unrivalled performance in extreme spatial field confinement [35-37]. The smallest demonstration of mode volume so far has been in picocavities, where the electromagnetic field is ultimately confined around a single metallic atom [9,38,39]. By using molecular monolayers, or atomically thin layers, so-called nanocavities can be routinely achieved, i.e., mode volumes $\leq 1 / 10^{5}$.

In order to hybridize such gap modes as exist in vertically assembled NPoM structures with a photonic crystal cavity, one would require a confined photonic mode with the main component of the electric field pointing along the vertical direction. This requires a photonic bandgap for transverse magnetic (TM) modes, which can be achieved by drilling holes in a thick nanobeam with a high index of refraction $[40,41]$. In this work, we introduce a novel class of hybrid cavity resulting from the hybridization between a metallic nanoparticle and a photonic crystal cavity in a high-index nanobeam that supports a highly confined TM mode at $\lambda_{c} \approx 700 \mathrm{~nm}$. We show that the upper surface of the photonic crystal can act as a low-reflectivity mirror when the metallic nanoparticle is placed on top of it. For nanoparticle-mirror gaps $(d)$ around $1 \mathrm{~nm}$ and through numerical calculations of the LDOS, we observe a strong reduction of the mode volume without a significant impairment of the $Q$ factor of the photonic cavity. Compared to the Purcell factors acquired with feasible gaps $(d \approx 10 \mathrm{~nm})$ for hybrid plasmonic-photonic configurations obtained from TE photonic modes, we reach an improvement of 1 order of magnitude. We also discuss the possible experimental design, which would require the use of materials with high index of refraction $(n>3)$ and negligible losses at visible and near-infrared spectral ranges, with gallium phosphide as a feasible candidate.

\section{DESCRIPTION OF THE HYBRID SYSTEM}

The system under study in this work is schematically depicted in the top panel of Fig. 1 [Fig. 1(a)]: a metal nanoparticle is placed on top of a one-dimensional photonic crystal cavity created in a high-index dielectric nanobeam. These two elements are depicted separately in Figs. 1(b) and 1(c). The goal of the hybridization is to improve the quality factor of the plasmonic nanoparticle by suppressing scattering pathways as a result of the photonic bandgap of the dielectric cavity, and to simultaneously decrease the mode volume of the dielectric cavity by squeezing the field with the metal nanoparticle. Note that, unlike in the standard NPoM configuration where the mirror is a metallic surface, in the proposed hybrid structure, it is the upper interface of the photonic crystal that acts as a mirror. This means that a high index of refraction is required to increase the reflectivity of the interface and boost the light confinement in the spacing gap. Moreover, in the current scheme, extreme light confinement will take place for the electric field pointing from the upper interface toward the metal nanoparticle. This means that the dielectric cavity must operate for TM polarization (or odd-parity modes in the context of photonic crystal slabs $[42,43])$. Obtaining odd-parity photonic bandgaps is not easy in thin semiconductor nanobeams drilled by holes, which usually tend to have bandgaps for transverse-electric (TE) modes $[42,44]$. However, by using thick substrates made of high-index dielectric materials (such as silicon at telecom wavelengths), the realization of high- $Q$ cavities for odd (or TM) modes by drilling holes becomes feasible $[40,41]$. Nevertheless, in order to operate at visible or near-infrared wavelengths, where many applications in the contexts of cavity quantum electrodynamics can be found, silicon is not allowed, and there are not so many transparent materials with a large index of refraction. Recently, gallium phosphide has been suggested as an interesting optical material for nonlinear [45] or optomechanical [46] applications. Interestingly, it shows a large refractive index $(n>3)$ and is transparent at wavelengths over $\lambda=570 \mathrm{~nm}$. For those reasons, this is the material that we have chosen to implement the photonic crystal cavity. In order to evaluate the effects of the dielectric substrate on the optical properties of the metal nanoparticle (when placed in its close proximity), we also consider the intermediate system depicted in Fig. 1(d). The analysis of this structure will allow us to separate the effects induced by the nonstructured dielectric substrate, merely acting as a "bad" mirror, and the whole perforated cavity having a bandgap for TM-polarized light.

\section{METHODS}

The results are calculated by means of the finite element method (FEM), implemented in the commercial software 

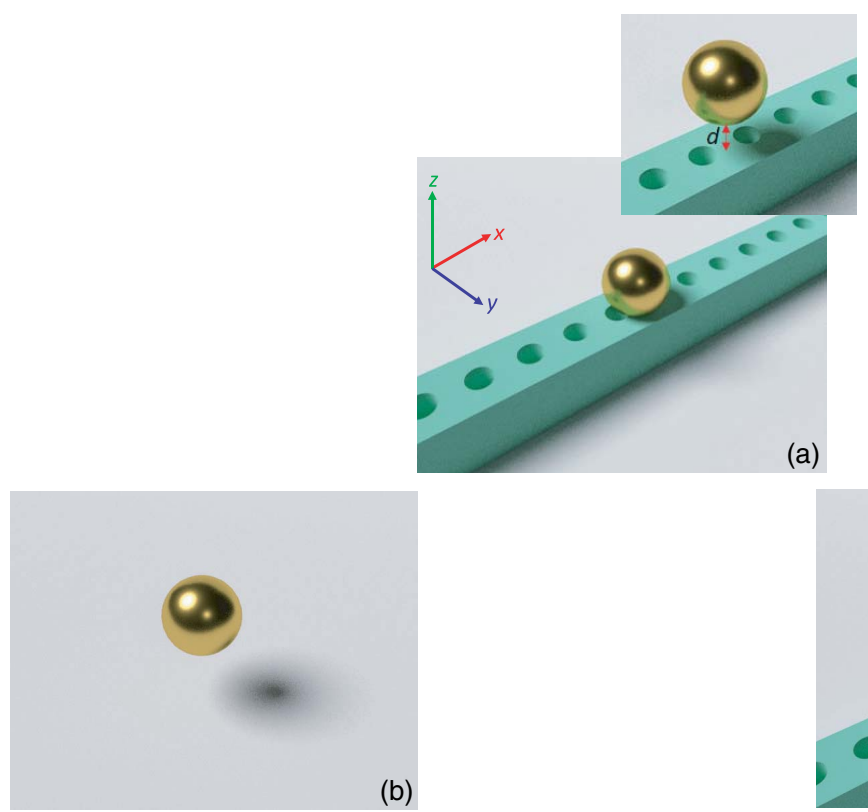

(a)

(b)
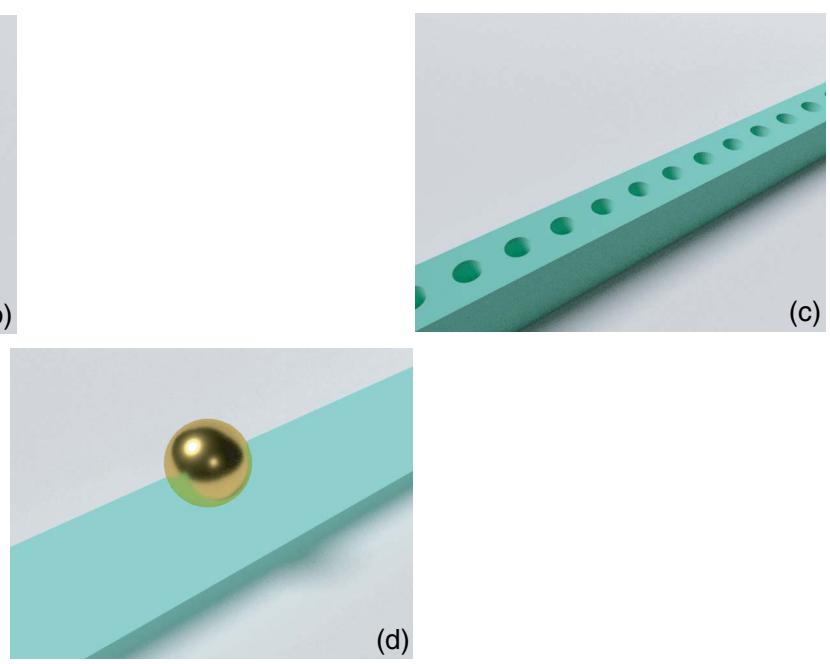

Fig. 1. Conceptual scheme of the hybrid system under study. (a) A metallic nanoparticle is placed on top of a dielectric cavity. Both structures are spaced by a tiny gap of thickness $d$, shown as an inset. The isolated systems are a (b) metallic nanoparticle, in this case a gold nanosphere, and (c) a photonic crystal cavity having a photonic bandgap for TM modes. (d) An intermediate system arises when the metal nanoparticle is placed on top of a nonstructured dielectric medium.

COMSOL Multiphysics [47]. Using the Radio Frequency Module in COMSOL, the Maxwell equations together with the boundary conditions are solved in the frequency domain. The optical constants for gold nanoparticles are taken from Ref. [48]. The refractive index for gallium phosphide $(\mathrm{GaP})$ is $n=3.49$. To rigorously determine the LDOS, the structure is illuminated by an electric point-dipole source, whose dipole moment is considered along the $z$ axis (along the axis connecting the sphere and the cavity). The normalized LDOS is defined as

$$
\text { LDOS }=\frac{P_{\text {rad }}+P_{\text {loss }}}{P_{\text {rad vacuum }}},
$$

where $P_{\text {rad }}$ is the radiative power density and $P_{\text {loss }}$ is the total power loss density. The numerator corresponds to the radiative and nonradiative power emitted by the dipole coupled with the structure. However, the denominator contains the radiative power emitted by the dipole in a vacuum. The dipole is located at the center of the structure and in the middle of the gap between the nanoparticle and the cavity; this means $0.5 \mathrm{~nm}$ below the nanoparticle and $0.5 \mathrm{~nm}$ above the surface of the beam. The hybrid geometry is surrounded by a cylindrical air region of radius $1.72 \mu \mathrm{m}$. An additional smaller cylinder with a radius of $1.11 \mu \mathrm{m}$ and made of air is placed in the center of the larger cylinder. The scattered power $\left(P_{\text {rad }}\right)$ is calculated at the boundaries of the smaller cylinder, whereas the total power loss density is obtained by means of the volume integration of the losses in the metallic nanoparticle. The dipole source is surrounded by a sphere with a diameter that is equal to the gap size $(d=1 \mathrm{~nm})$ to ensure a sufficient fine grid in close proximity to the dipole source. The mesh of the surrounding air medium is chosen to be smaller than $150 \mathrm{~nm}$, and that of the cavity and particle is smaller than $45 \mathrm{~nm}$. The mesh of the sphere surrounding the dipole source is smaller than $1.2 \mathrm{~nm}$. The cylindrical region of air is surrounded by a perfectly matched layer (PML) with a thickness of $500 \mathrm{~nm}$. A graphical description of the COMSOL model can be found in Appendix A. The mode profiles are calculated with the eigenmode solver of COMSOL Multiphysics. Through these near-field plots we can see the electric field distribution in the structure for the eigenfrequencies of interest. It is remarkable to clarify that the same mesh is used for the LDOS and eigenmode calculations and for the different analyzed structures: bare nanoparticles, bare cavity, and hybrid. In all the cases, the same geometry is considered, and only optical constants of the materials are changed appropriately. For the near-field map corresponding to the isolated sphere, the structure is illuminated with a plane wave propagating along the $x$ axis and linearly polarized along the $z$ axis. 
The reason is that with this calculation, from the scattered field distribution, we are also able to identify the excited mode (dipolar electric), and the computation is faster than calculating the eigenfrequency values. For this simulation, the nanosphere is located in a homogeneous spherical air region of radius $700 \mathrm{~nm}$. The PML thickness corresponds to $200 \mathrm{~nm}$. To ensure numerical convergence of the results, the tetrahedral mesh is chosen to be sufficiently fine. Thus, the mesh of the nanosphere is smaller than $10 \mathrm{~nm}$ (for a graphical description of the simulated geometry, see Appendix A). The same model was used to attain the absorption, scattering, and extinction cross sections of the bare nanoparticles. In Appendix B, we present some convergence tests performed to verify the accuracy of our simulations.

\section{RESULTS}

We start by considering the electromagnetic response of an isolated metallic nanosphere, which for small radius exhibits a dipolar plasmonic response at visible wavelengths. In Fig. 2(a), we show the calculated normalized LDOS for a gold nanosphere of radius $R=40 \mathrm{~nm}$, which displays a dipolar electric resonance at $\lambda_{\mathrm{NP}} \approx 500 \mathrm{~nm}$. In analogy to Ref. [16], LDOS values are normalized to the LDOS in a vacuum at the same wavelength, with LDOS containing radiative as well as nonradiative contributions. It is worth noting that, although in the text we write LDOS, rigorously, this magnitude corresponds to partial LDOS (PLDOS) and not to LDOS, as the emitter is not only sensitive to the intensity of the available modes but also to their polarization. A dipole aligned along the $z$ axis will interact most strongly with those modes where the electric field is polarized in the same direction [49]. The optical quality factor $Q_{\mathrm{NP}}=7.91$ (retrieved by means of a Fano line shape fitting; in Appendix C, an explanation is provided of the performed fittings in the work, together with their equations) and the normalized mode volume $V_{\mathrm{NP}}=1.0 \times 10^{-6}$ are shown in the inset. While only LDOS is a rigorous physical quantity, it has become practice in the field to interpret near-Lorentzian (a)

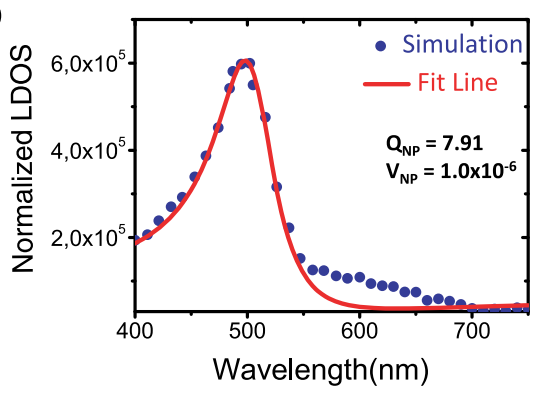

(c)

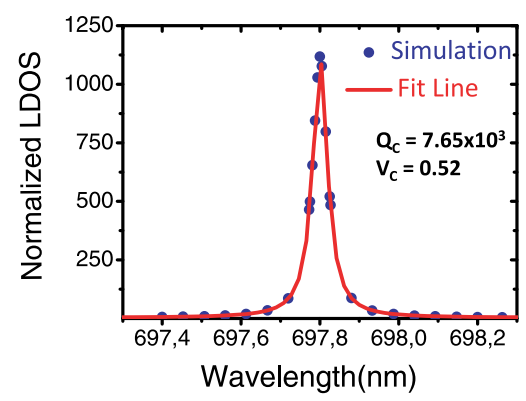

(e)

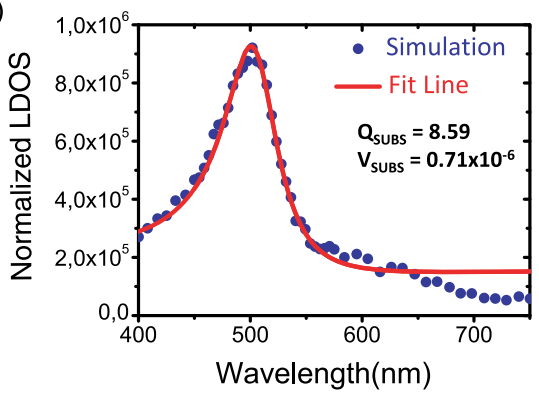

(b)
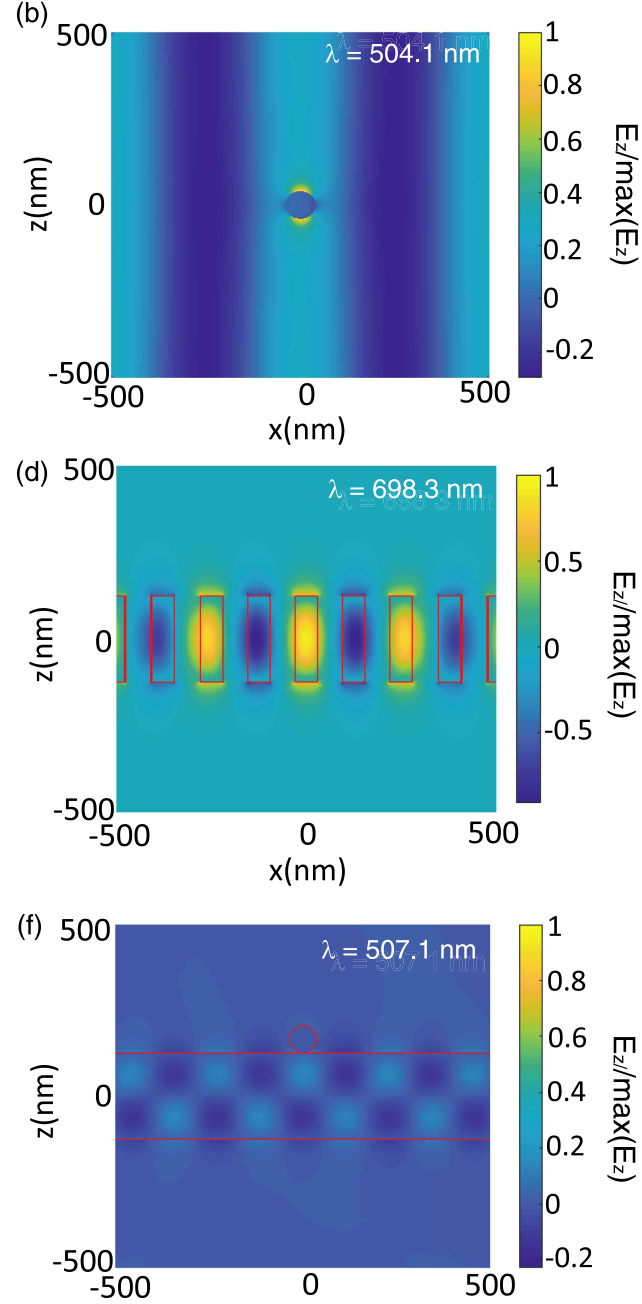

Fig. 2. Simulation results of the different building blocks of the hybrid cavity. (a) Normalized LDOS, $Q$, and $V$ and (b) mode profile $(z-x$ crosscut) of the $z$ component of the electric field normalized to its maximum value for a gold nanoparticle of radius $R=40$ nm; (c), (d) are like (a), (b) but for the dielectric gallium phosphide photonic crystal cavity described in the main text; (e), (f) are like (a), (b) but assuming that the nanosphere $(R=40 \mathrm{~nm})$ is placed on top of a nonstructured gallium phosphide dielectric slab (width $w=200 \mathrm{~nm}$ and thickness $t=250 \mathrm{~nm})$. 
LDOS peaks in terms of $Q$ and $V$, simply by reading of $Q$ from the line shape and inverting peak LDOS into a volume via Eq. (1). We follow this practice in this work, always quoting LDOS, $Q$, and derived $V$ together. This practice is adopted only for qualitative practical comparison with other resonators and does not imply any endorsement of the concept of Purcell factor and mode volume for open, lossy, or multimode resonators. The term "mode volume" here is not used as an endorsement of the validity of this concept per se for plasmonics, and the term also does not indicate that we employed a quasi-normal mode formalism. Noticeably, $V$ becomes very small; as expected from plasmonic cavities, far smaller than the diffraction limit. The extreme confinement is clearly appreciated in Fig. 2 (b), which depicts the near-field map corresponding to the $z$ component of the electric field under resonance, with linearly polarized light (along the $z$ axis) propagating along the $x$ axis. This figure corroborates the dipolar character of the excited mode at resonance in Fig. 2(a).

When the photonic crystal cavity is considered, the $Q$ versus $V$ situation is reversed. Our dielectric cavity consists of a gallium phosphide ( $\mathrm{GaP}$ with refractive index $n=3.49$ ) nanobeam of width $w=200 \mathrm{~nm}$ and thickness $t=250 \mathrm{~nm}$ drilled with circular holes. To build the cavity, we calculate the parameters to get a TM bandgap around $\lambda \approx 700 \mathrm{~nm}$ (see Appendix D, where we represent the band diagram for the photonic crystal and the defect), which happens for a period $P_{M}=165 \mathrm{~nm}$ and hole radii of $R_{M}=43.7 \mathrm{~nm}$. We choose this wavelength because, as shown in previous works [16], the nanoparticle resonance must be blueshifted regarding that of the photonic cavity to improve the performance of the hybrid cavity with respect to the bare components. Nevertheless, the resonance wavelength could be either red- or blue-shifting by changing the parameters defining the photonic crystals. With the previous dimensions, we form two TM mirrors with 10 holes each at every side of the cavity. The photonic cavity is subsequently formed by adiabatically changing the dimensions of the mirrors when moving toward the cavity center, where the nanoparticle will be placed. In particular, we reduce the period and hole size of the photonic crystal during seven holes at each side of the cavity by means of a quadratic adiabatic transition down to $P_{D}=138.6 \mathrm{~nm}$ and $R_{D}=36.7 \mathrm{~nm}$, respectively $[40,41]$. This leads to a confined TM mode with a large $Q$ factor as shown in Fig. 2(c), which depicts the obtained normalized LDOS for the isolated photonic cavity, calculated by considering the illumination of an electric dipole placed $0.5 \mathrm{~nm}$ above the cavity. From the fitting of the data to a Lorentzian line shape (see Appendix $\mathrm{C}$ for an explanation about the fitting and their equations), the $Q$ factor and normalized mode volume $V$ values are extracted. In this case we obtain a large $Q$ factor $\left(Q_{C}=7.65 \times 10^{3}\right)$, which could be further engineered to values even above $10^{5}$, and a diffraction-limited normalized volume $V_{C} \approx 1$. Looking at the mode profile of the $z$ component of the electric field distribution in the cavity at resonance [see Fig. 2(d)] (z-x crosscut of the cavity design corresponding to the length-thickness plane at the middle of the beam width), we can observe that it has evanescent tails of $E_{z}$ on top of the upper interface, which may couple to the evanescent field perpendicular to the nanosphere surface [Fig. 2(b)] and thus give rise to the pursued hybridization of the plasmonic and photonic resonances. The field distribution, calculated by using the COMSOL Multiphysics eigenmode solver, corresponds to the fundamental mode. Henceforth, all the near-field maps shown in this work are obtained using this methodology. The tiny oscillations in Figs. 2(a) and 2(c) are related with internal reflections with the PMLs in the implemented simulation box and correspond to numerical noise. Since the spectral position of the peak and its width as well as convergence are achieved, we assume these numerical artefacts are small enough to be considered in our main results.

In order to estimate the effect of the dielectric layer acting as a low-reflectivity mirror, we also simulate the response of the metallic nanosphere when located on the top of a nonstructured GaP nanobeam (width $w=200 \mathrm{~nm}$ and thickness $t=250 \mathrm{~nm}$ ) to determine the influence of the dielectric substrate on the electromagnetic behavior of the gold nanoparticles. In other words, we simulated the same geometry as in the case of the hybrid cavity (below) but without holes. The gap between the metallic sphere and the dielectric slab corresponds to $d=1 \mathrm{~nm}$, a value that is kept in all our calculations. For this configuration, the $Q$ factor $\left(Q_{\text {SUBS }}=8.59\right)$ and normalized mode volume $V\left(V_{\text {SUBS }}=0.71 \times 10^{-6}\right)$ are similar to those obtained for the isolated sphere. This evidences that the $\mathrm{GaP}$ substrate behaves as a "bad" mirror as well as the necessity of including the photonic crystal to build up the hybrid system.

To form the hybrid cavity, we place the gold nanosphere on top of the photonic crystal beam [Fig. 1(a)] keeping $d=1 \mathrm{~nm}$. In real experiments, this spacer would be filled by a self-assembled monolayer (SAM). To understand how the detuning between the cavity and nanosphere resonant wavelengths affects the results, different radii for the metallic nanosphere are considered, varying from $R=30$ to $70 \mathrm{~nm}$ in $10 \mathrm{~nm}$ steps. In Figs. 3(a) and 3(b), the normalized LDOS spectra, the $Q$ factor, and the normalized mode volume values $V$ for the different analyzed hybrid systems are shown. In Figs. 17(a) and 17(b) of Appendix E, the same parameters (normalized LDOS, $Q$ factor, and $V$ ) are represented for the isolated gold nanoparticles, i.e., without considering the cavity. Again, the LDOS is obtained by considering the illumination of an electric dipole placed $0.5 \mathrm{~nm}$ above the cavity, whose dipole moment is oriented along the $z$ axis. The parameters of the hybrid, $Q_{\mathrm{HYB}}$ and $V_{\mathrm{HYB}}$, are retrieved through the fitting of the LDOS to a Fano line shape. By comparing the results for the hybrid and the bare systems, it is observed how the hybrid $Q_{\mathrm{HYB}}$ and $V_{\mathrm{HYB}}$ take intermediate values between those of the photonic cavity and the nanoparticle. This means that, as expected, the hybrid cavity enables small volumes (order $10^{-3}$ ) below the diffraction limit whereas $Q_{\text {НYв }}$ remains relatively high (order $10^{3}$ ). This is an indicator of the hybridization of the cavity and nanoparticle responses. The hybridization effect is clearly observed in Fig. 3 (c), where we represent the mode profile ( $z-x$ crosscut) of the $z$ component of the electric field for a nanosphere-based hybrid cavity with $R=40 \mathrm{~nm}$. The extreme concentration of the electric field in the gap, mimicking what takes place in standard NPoM systems, is shown in the inset. In Fig. 3(a) we also observe that as the gold nanoparticle radius increases, its resonant wavelength is redshifted, and the detuning between the metallic 
(a)

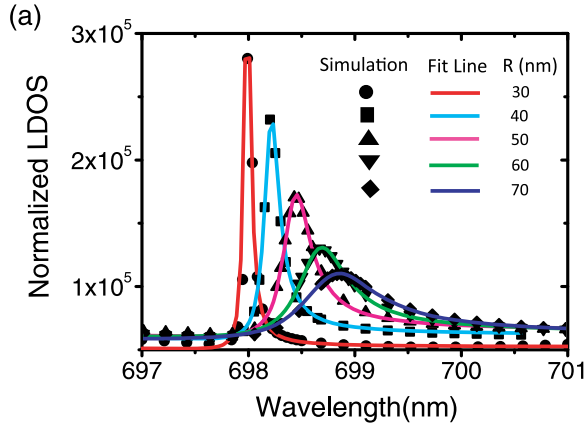

(b)

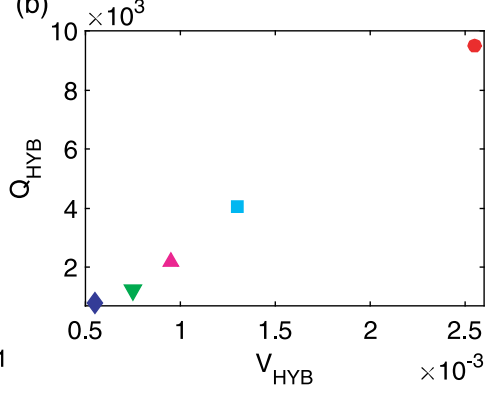

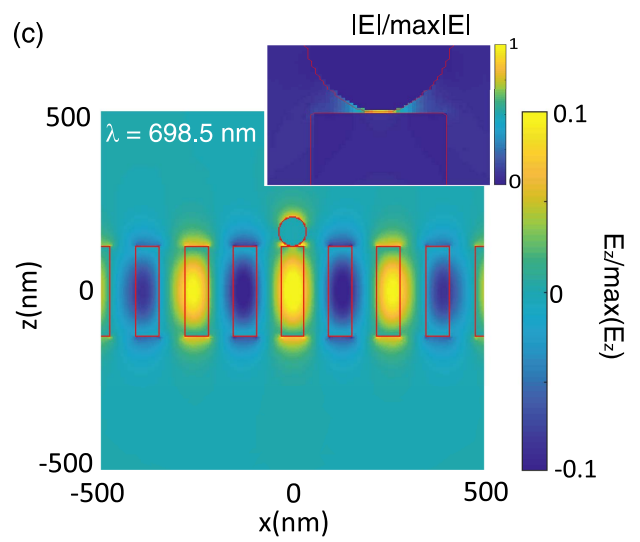

Fig. 3. Simulation results of the hybrid cavity as a function of the gold nanosphere radius. (a) Normalized LDOS and (b) quality factor and normalized mode volume for the hybrid system constituted by the cavity beam and a gold nanoparticle of radius varying from $R=30$ to $70 \mathrm{~nm}$. The sphere is separated from the cavity by a gap of $d=1 \mathrm{~nm}$. (c) Mode profile ( $z-x$ crosscut) of the $z$ component of the electric field normalized to its maximum value. Note that the scale is saturated to improve visualization. The electric field amplitude in the gap $\left(\left|\mathbf{E}_{\text {gap }}\right|\right)$ normalized to its maximum value is represented in the inset for an $R=40 \mathrm{~nm}$ nanosphere-based hybrid cavity.

nanosphere and the nanobeam cavity decreases. As a result, the resonances are broadened, and both the $Q$ factor and normalized mode volume decrease. In fact, the results for $R=30 \mathrm{~nm}$ correspond to $Q_{\mathrm{HYB}}=0.95 \times 10^{4}$ and $V_{\mathrm{HYB}}=2.6 \times 10^{-3}$, while for $R=70 \mathrm{~nm}$, they are $Q_{\mathrm{HYB}}=0.8 \times 10^{3}$ and $V_{\mathrm{HYB}}=0.6 \times 10^{-3}$. We also performed simulations for smaller spheres (not shown). In particular, for $R=20 \mathrm{~nm}$ we get $V_{\mathrm{HYB}} \approx 10^{-2}$, which means that strong subwavelength spatial confinement arising from the plasmonic response is eventually lost.

We also consider different shapes of the metallic nanoparticle to be coupled to the photonic crystal cavity: a nanocube and a nanoellipse (see Fig. 4). In Figs. 17(c) and 17(d), we show the results for the LDOS, $Q$ factor, and $V$ corresponding to the bare nanocube and nanoellipse, respectively. Metal nanocubes, which like nanospheres can be easily built by chemical procedures, are also able to play the role of the plasmonic particle in NPoM configurations $[37,50]$. Remarkably, the results in terms of $Q$ and $V$ are similar to the case of the nanosphere previously presented in Fig. 3. The nanoellipse, which also clearly shows the hybridization between the plasmonic and photonic resonances, is included to mimic the effect of a sharp metallic tip that may be placed near the SAM as in other previous experiments for extreme light-matter interaction in sub-nm gaps [51]. This demonstrates that our proposed design is versatile and capable of functioning as a hybrid for different kinds of metallic nanoparticles.
To gain further insight into the hybridization of the metallic nanoparticle with the dielectric cavity, in Fig. 5 we plot the mode profile of the amplitude of the electric field $(|\mathbf{E}|)$ for the $x-y$ crosscut (corresponding to the top surface of the cavity) at the position of the dipole $(0.5 \mathrm{~nm}$ above the cavity). The analysis is performed for the three different studied geometries (sphere, ellipsoid, and cube). For comparison, the field on the bare cavity is also included. Noticeably, when the metallic particle is considered, a subwavelength hot spot is observed just below the nanoparticle. This hot spot, which closely resembles the standard NPoM case, results from the squeezing of the evanescent vertical field in the photonic cavity by the plasmonic nanoparticle.

Besides the Purcell factor, it is also worthwhile to consider the radiative efficiency of the different hybrid modes. In the calculations above, we have considered both radiative as well as nonradiative contributions to the LDOS. In order to ensure an efficient excitation to the environment (bright hybrid mode), it is desirable that the radiative contribution dominates over the nonradiative one. We have performed a rigorous analysis of the quenching emission in Appendix F. In Fig. 18, we have included the contributions of the radiative and nonradiative power to the normalized LDOS for the different nanoparticle-based hybrid cavity configurations analyzed in the manuscript (nanosphere, nanocube, and nanoellipsoid). The radius of the nanosphere corresponds to $R=40 \mathrm{~nm}$. It is observed that, at the resonance wavelength, for the nanosphere, 
(a)

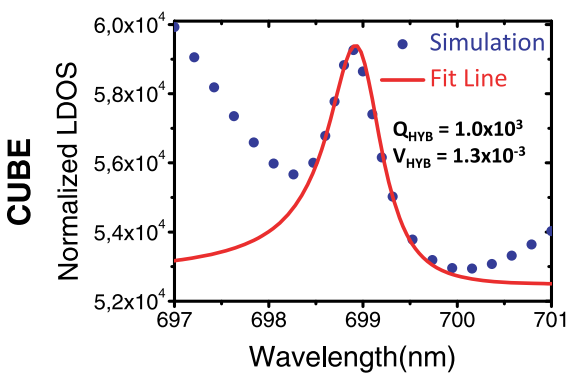

(c)

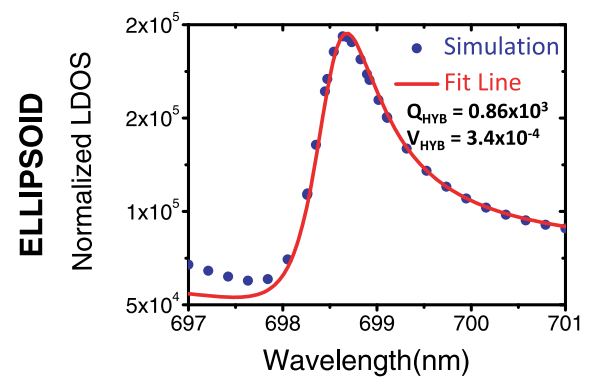

(b)

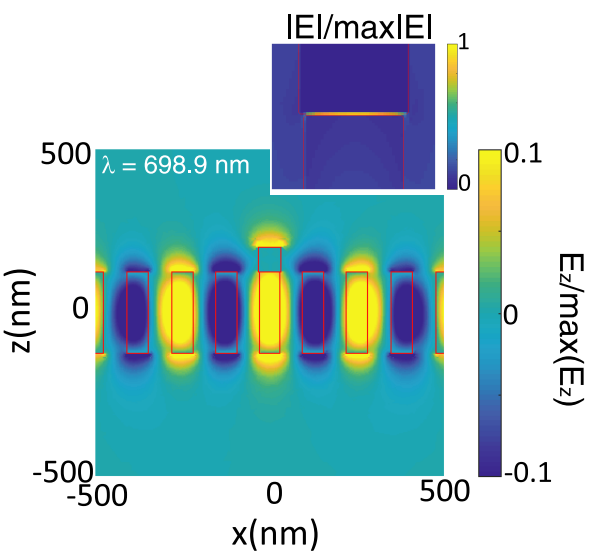

(d)

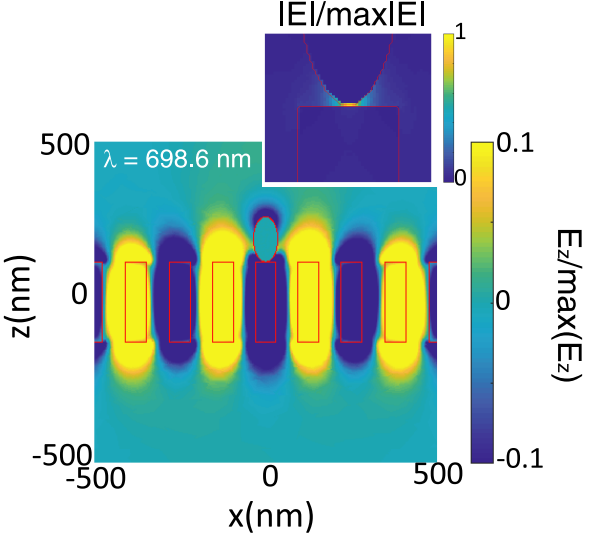

Fig. 4. Simulation results of alternative configurations for the hybrid cavity. (a) Normalized LDOS, quality factor, and normalized mode volume and (b) mode profile $(z-x$ crosscut) of the $z$ component of the electric field normalized to its maximum value. Note that the scale is saturated to improve visualization. The electric field amplitude in the gap $\left(\left|\mathbf{E}_{\text {gap }}\right|\right)$ normalized to its maximum value is represented in the inset for a nanocubebased hybrid cavity. The length side of the cube is $l=75 \mathrm{~nm}$. (c), (d) are like (a), (b) but for a nanoellipse-based hybrid cavity. The width and length of the ellipsoid are $w_{e}=40 \mathrm{~nm}$ and $l_{e}=70 \mathrm{~nm}$, respectively.
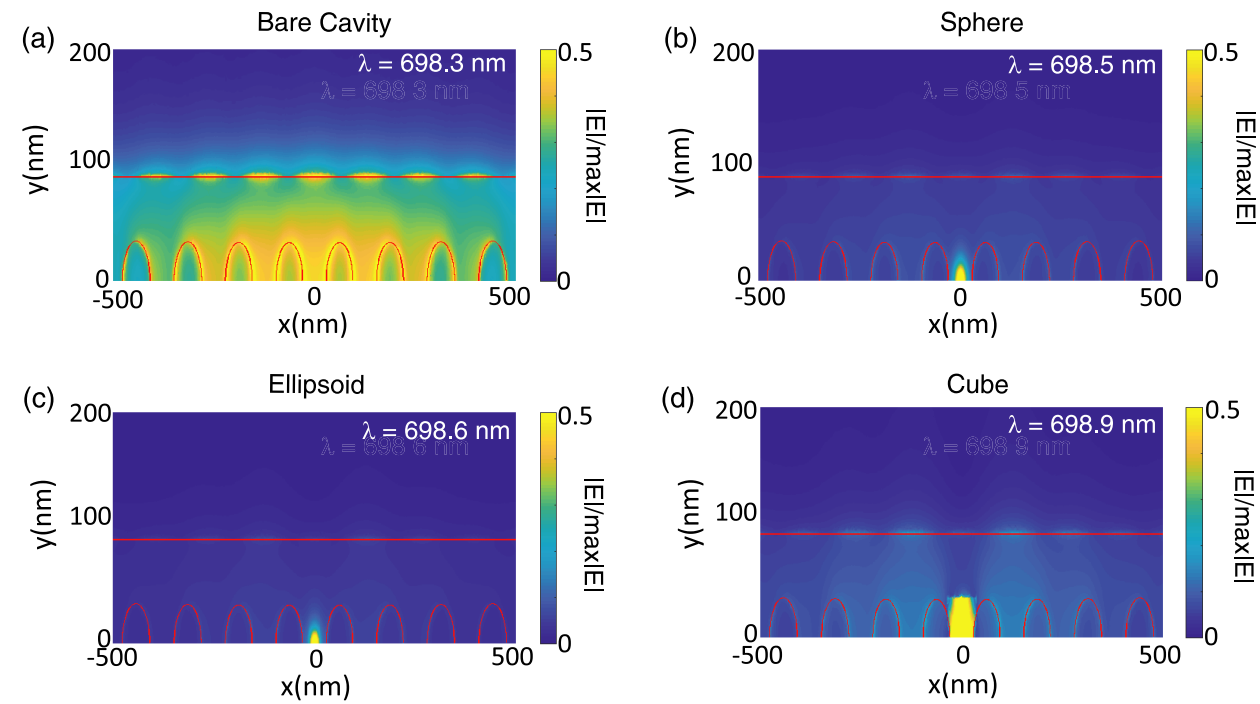

Fig. 5. Mode profiles of the electric field amplitude $|\mathbf{E}|$ corresponding to the $x$ - $y$ crosscut at the position of the dipole (0.5 nm above the cavity) normalized to their maximum values. The (a) bare cavity, (b) sphere, (c) ellipsoid, and (d) cube cases are shown. Note that the scale is saturated to improve visualization. 
the radiative power takes larger values than the nonradiative power, obtaining a ratio of radiative/nonradiative LDOS of 1.5 [see Fig. 18(b)], which is the largest of the three studied geometries. For the case of the ellipsoid, the radiative contribution is also larger than the nonradiative one. In fact, the ratio radiative/nonradiative LDOS takes a value of 1.3 at the resonance wavelength [Fig. 18(f)]. For the cube, the radiative power is small compared to the nonradiative power. The radiative efficiency for this shape is quite low [ratio radiative/nonradiative LDOS is 0.09 , Fig. 18(d)]. For that reason, spheres and ellipsoids are more promising structures, as they show high $Q$ factor and small $V$ values, but at the same time preventing of high emission quenching. This behavior can be explained attending to the hybridization of the TM fundamental mode of the cavity with the plasmonic mode excited in the NP. As the particle exhibits a more dipolar character, the hybridization between the modes of the cavity and the NP is larger, increasing the radiative power density with respect to the power loss density. However, it is worth remarking that, still for the cube case, in the current scheme, the hybrid mode could be easily excited using TM guide modes of the dielectric waveguide, as usually done in direct inline coupling of photonic crystal cavities. In Fig. 19, we represent the evolution of the radiative contribution to LDOS and the ratio between the radiative and nonradiative power as a function of the nanosphere radius in a nanosphere-based hybrid cavity. The chosen sizes are the same as in the manuscript $(R=30-70 \mathrm{~nm})$. It is observed that the radiative emitted radiation, and also, the ratio radiative to nonradiative emission increases with the decrease in the radius of the nanosphere. In Fig. 20, we show the LDOS of the hybrid NP-cavity configurations considering only the radiative contribution. Also, in Fig. 20(d), we compare the $Q$ and $V$ values taking into account the total LDOS (radiative + nonradiative), and only the radiative contribution to LDOS for the nanosphere/nanocube/nanoellipsoid-cavity systems. We could conclude that similar $Q_{\mathrm{HYB}}$ and $V_{\mathrm{HYB}}$ values are obtained. This suggests that our proposed hybrid systems are able to improve the $Q$ and $V$ values with respect to the bare components (nanoparticles and cavities) when only the radiative part is considered. The radiative and nonradiative contributions to LDOS for the bare nanoparticles are shown in Fig. 21. From those plots, it can be observed that the dominant contribution is the nonradiative part, as it was expected for a metallic NP illuminated by a dipole in its proximity (distance from the dipole to the metallic NP $d=0.5 \mathrm{~nm}$ ). To understand the influence of the dipole source illumination with respect to that corresponding to a plane wave, we represent in Fig. 22 the absorption, scattering, and extinction cross sections spectra for the bare nanosphere, nanocube, and nanoellipsoid. To understand which modes are responsible for the resonances, we have performed a multipolar decomposition for the nanosphere $(R=40 \mathrm{~nm})$, nanocube, and nanoellipsoid. The results are found in Figs. 22(b), 22(d), and 22(f). It is observed that for all the analyzed geometries, the resonance has dipolar electric character. However, contributions of higher-multipolar orders may appear when the NPs are illuminated by an electric dipole, instead of a plane wave (as it is done for attaining the multipolar decomposition). The excitation of the higher-multipolar orders is responsible for the discrepancies observed between the couple harmonic oscillator $(\mathrm{CHO})$ model and the numerical simulations, explained in the next section.

\section{COUPLED HARMONIC OSCILLATOR MODEL}

Recently, it has been shown in a theoretical work by Doeleman et al. [10] and confirmed in a numerical study in Ref. [16] that the behavior of hybrid plasmonic-photonic resonator systems can be accurately predicted by simple analytical modeling of antenna and cavity as a set of coupled harmonic oscillators, driven by a dipole source that represents an emitter. This $\mathrm{CHO}$ model was verified to hold quantitatively for a large class of hybrid plasmonic-photonic resonators and predicts hybrid resonator performance by considering as input exclusively the properties of the bare antenna (scattering properties, LDOS enhancement in absence of the cavity) and cavity (mode volume, $Q$ ). In particular, the class of systems for which the model was verified includes photonic crystal nanobeams supporting TE confined modes coupled to plasmonic dipolar antennas (such as bow-tie antennas). The physics underlying the model is the assumption that antenna and cavity are weakly coupled, and therefore the analysis of the antenna response can be properly described through its induced dipole moment. Here we verify whether the structures studied in this work, with their extremely small gaps, can be similarly treated.

To recapitulate the modeling approach, the total LDOS $\left(\mathrm{LDOS}_{\text {tot }}\right)$ for such hybrid systems can be expressed as [10]

$$
\mathrm{LDOS}_{\mathrm{tot}}=1+\frac{6 \pi \epsilon_{0} c^{3}}{\omega^{3} n} \operatorname{Im}\left(\alpha_{H} G_{\mathrm{bg}}^{2}+2 G_{\mathrm{bg}} \alpha_{H} \chi+\chi_{H}\right),
$$

where $n$ is the refractive index of the background medium, $c$ is the speed of light, and $\omega$ is optical frequency. The $\operatorname{LDOS}_{\text {tot }}$ is determined by the interference of three terms, each of which combines response functions of antenna and cavity, dressed by the multiple scattering interactions between them. The subscripted quantities $\alpha_{H}=\alpha /(1-\alpha \chi)$ and $\chi_{H}=\chi /(1-\alpha \chi)$ represent the hybrid polarizability of the antenna (antenna polarizability modified by the presence of the cavity) and the hybrid cavity response function as perturbed by the presence of the antenna, respectively. These hybrid quantities are in turn set by the bare antenna polarizability $\alpha$, which sets the antenna scattering and extinction cross sections in the absence of the cavity structure and the bare cavity response function $\chi$. Equation (3) should match the LDOS provided by just the bare antenna if the cavity terms are removed (setting $\chi=0$, also leading to $\alpha_{H}=\alpha$ ). Given that $\alpha$ is already fixed by the antenna scattering and extinction cross section, this matching of LDOS is accounted for by $G_{\mathrm{bg}}$, which in a point dipole model is interpreted as the coupling between antenna and source as given by the Green's function in the surrounding background medium. In a similar spirit, in absence of the antenna $\alpha=0$, the LDOS in Eq. (3) should match the bare cavity LDOS. This sets the Lorentzian response function of the bare cavity $\chi=\frac{1}{\epsilon_{0} V_{C}} \omega^{2} /\left(\omega_{C}^{2}-\omega^{2}-i \omega \kappa\right)$ that has resonance frequency $\omega_{C}$ and damping rate $\kappa=\omega_{C} / Q$ matching the resonance and $Q$ of the unperturbed cavity, and in which the effective mode volume $V_{C}$ appears such that Eq. (3) matches the 
Purcell factor of the cavity at the assumed location of the emitter.

In order to investigate the agreement between our COMSOL simulations and the $\mathrm{CHO}$ model, we performed additional simulations on the antennas discussed above, again using COMSOL Multiphysics, to calculate the scattering and extinction cross sections of each antenna in absence of the cavity and by using plane wave excitation. Within the assumption of dipolar scattering, the obtained frequency-dependent scattering and extinction cross sections can be used to obtain the antenna polarizability by inversion of the relations [52]

$$
\sigma_{\text {scat }}=\frac{\omega^{4}}{6 \pi \epsilon_{0}^{2} c^{4}}|\alpha|^{2}, \quad \sigma_{\text {ext }}=\frac{\omega}{\epsilon_{0} c n} \operatorname{Im}(\alpha) .
$$

Next, once we have determined $\alpha$ from the far-field scattering properties, we determine $G_{\mathrm{bg}}$ (which is approximately real-valued in the near field) from COMSOL calculations of the total LDOS provided at the emitter by inverting Eq. (3) (setting the cavity terms to 0 ). For a benchmarking of this procedure for dipolar gap antennas, we refer the reader to Refs. $[10,16]$. We note that the inversion of scattering properties into polarizability only works in frequency domains where the cross sections are in magnitude within the constraints of the unitary limit that provides an upper bound on extinction of dipolar scatterers $\left(\sigma_{\text {ext }} \leq 3 / 2 \pi \lambda^{2}\right)$. While some of the antennas at hand do not satisfy this constraint at their resonance frequency, the antenna-cavity hybrids operate significantly to the red of the antenna resonance frequencies. At the relevant operation frequencies, all the antennas show far-field scattering properties well described as electric dipole scattering.

In Fig. 6 we show the LDOS calculated from the $\mathrm{CHO}$ model, evaluating Eq. (3) with parameters for the bare constituents extracted from the COMSOL simulations. We find that the $\mathrm{CHO}$ model (blue lines in Fig. 6) predicts Fano line shapes in LDOS for all the hybrids at hand that are qualitatively similar to those calculated with full-wave simulations of LDOS for the full hybrid system (green curves/symbols). Quantitatively, the $\mathrm{CHO}$ prediction significantly overestimates the maximum achievable LDOS of the hybrid systems by a factor of 10-50. In addition, the full-wave numerical simulations are shifted significantly less than the $\mathrm{CHO}$ model at the resonance wavelength; they feature a different degree of broadening, and they generally have a poorer contrast between peak LDOS and background LDOS (LDOS a few linewidths away from the Fano resonance). This should be contrasted to earlier findings by Palstra et al. [16] wherein the very same procedure gave an excellent quantitative match between the $\mathrm{CHO}$ semianalytical model and full-wave simulations of hybrid LDOS, in the case of TE-cavity modes in photonic nanobeams coupled to dipolar gap antennas.

The discrepancy between the $\mathrm{CHO}$ model and the full-wave results points to the fact that the NPoM-cavity hybrids operate in a qualitatively different regime than previously reported hybrid plasmonic-photonic resonators constructed from dipole antennas and photonic crystal nanobeams. At the extremely small gaps considered in this work, the antennas do not respond with only their electric dipole mode as assumed in the $\mathrm{CHO}$ model, and instead high multipole orders in their excitation also contribute. Even without examining the hybrid LDOS spectra, the failure of the dipole model is already evident from the anomalously small frequency shift induced in the cavity mode by the antennas. In cavity perturbation theory, a seminal result due originally to Waldron and Bethe is that the complex resonance frequency of the unperturbed cavity $\tilde{\omega}_{c}=\omega_{0}+i \kappa / 2$, where as before $\kappa$ is the FWHM of the unperturbed cavity resonance, will shift by $\Delta \tilde{\omega}$ following

$$
\frac{\Delta \tilde{\omega}}{\tilde{\omega}}=-\frac{\alpha}{2 V_{C}},
$$

where $V_{C}$ is the mode volume of the unperturbed cavity [53-57]. In more recent literature it was shown that this expression generalizes to a wide class of photonic resonances, provided that the mode volume $V_{C}$ is generalized through quasinormal mode concepts [58-62]. In this perturbation formula the real part of antenna polarizability $\alpha$ results in a frequency shift and the imaginary part in additional resonance broadening. It can be analytically verified that the $\mathrm{CHO}$ model intrinsically reproduces exactly this well-known expression. In other words, the $\mathrm{CHO}$ model predicts the hybrid LDOS Fano resonance to occur at the perturbed cavity resonance predicted by Eq. (5). Comparing the resonance shifts between COMSOL simulation and the $\mathrm{CHO}$ model in Fig. 6 thus directly shows
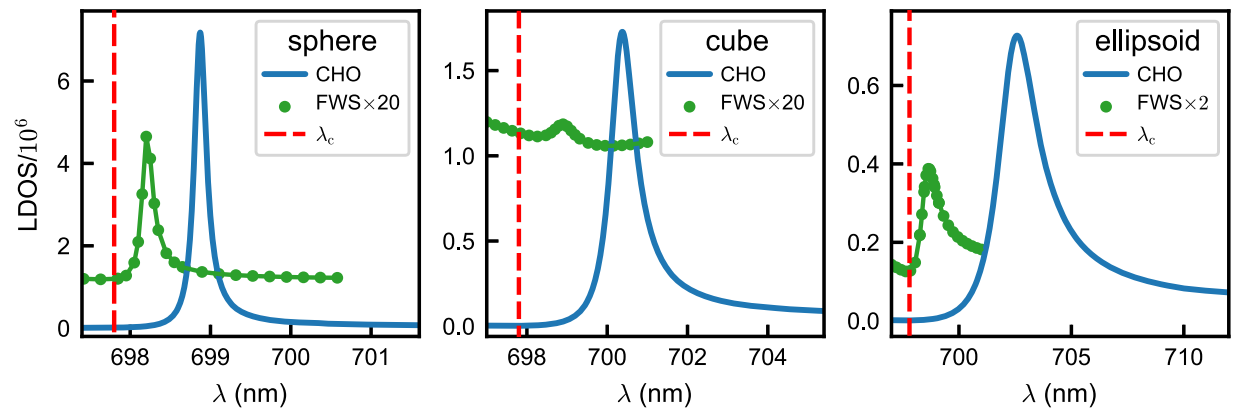

Fig. 6. Comparison of the LDOS as a function of the wavelength for the three hybrid systems considered. The green line shows the results of the full-wave simulations (labeled FWS) discussed above. The blue curve shows the LDOS as calculated with Eq. (3) (labeled CHO for coupled oscillator model). The resonance frequency of the cavity in absence of the nanoparticle is indicated as $\lambda_{c}$ (dashed vertical lines). The full-wave simulations have been scaled as indicated. 
that in all the cavity-antenna constructs the frequency shifts are far smaller than what would be expected on the basis of perturbation theory and the antenna far-field scattering properties that are captured by the polarizability $\alpha$. These discrepancies can be directly ascribed to the fact that Waldron's formula assumes the perturbation to be placed in a part of the cavity field where the gradient is small, so that the perturber provides an essentially quasistatic dipolar response. Instead, the field plots directly show very strong field gradients in/at the antenna. Such gradients, and particularly the extreme gap fields, are only supported by virtue of retarded and multipolar contributions [60]. We thus conclude that the NPoM-inspired hybrids proposed in this work are qualitatively distinct from the previously reported microdisk and nanobeam resonator hybrids due to significant multipolar corrections.

\section{DISCUSSION}

It is useful to provide a perspective on the achievable $Q$ and $V$ values in the proposed NPoM-inspired hybrids. Figure 7 depicts the $Q$ versus $V$ values for the different structures under study in this work. Through this diagram it is possible to compare the hybrid systems and the isolated constituents in correspondence with Ref. [16]. As expected, the hybrid systems are in the intermediate regime, taking $Q$ and $V$ values between those of the photonic cavity and the plasmonic nanoparticle. Dashed diagonal lines show lines of constant Purcell factor. At the very small emitter-particle distances considered in this work, the LDOS values and $Q s$ for the bare nanoparticles and for the sphere on a nonstructured substrate are quite similar. Our hybrid cavities enable values of $F_{P}$ above $10^{5}$. For these extremely narrow gaps, the LDOS enhancement in terms of achievable peak LDOS is less advantageous than extrapolated from the $\mathrm{CHO}$ model. At the same time, the achievable LDOS

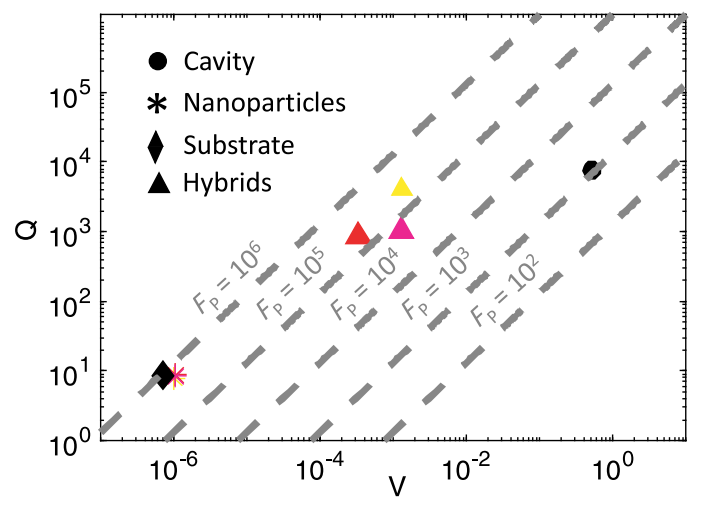

Fig. 7. Quality factors $Q$ and normalized effective mode volumes $V$ for bare metallic nanoparticles (asterisks of different colors according to the nanoparticle geometry: sphere, yellow; ellipsoid, red; and cube, magenta), the photonic crystal nanobeam cavity (circle), the spherical nanoparticle $(R=40 \mathrm{~nm})$ on a nonstructured gallium phosphide substrate (diamond), and the hybrids (triangles of different colors according to the geometry of the nanoparticle on the cavity: sphere, yellow; ellipsoid, red; and cube, magenta). The dimensions for the different nanoparticles are: sphere $(R=40 \mathrm{~nm})$, ellipsoid $\left(w_{e}=40 \mathrm{~nm}\right.$ and $\left.l_{e}=70 \mathrm{~nm}\right)$, and cube $(l=75 \mathrm{~nm})$. Diagonal dashed lines are lines of constant Purcell factor with value $F_{P}$ as labeled. values are higher than those that have been predicted for hybrids composed of standard TE-polarized photonic crystal nanobeam cavities and bow-tie antennas [16], for bow-tie gaps that are realistically achievable by lithography (limited to circa $15 \mathrm{~nm})$. To attain LDOS values in such TE hybrids based on bow-tie nanoantennas that are as high as we find in this work for NPoM-inspired hybrids would require bow-tie gaps below $5 \mathrm{~nm}$, which are challenging to achieve in a controllable fashion as discussed above.

\section{CONCLUSIONS}

In summary, we propose a novel design of hybrid photonicplasmonic cavities based on the combination of an NPoM plasmonic cavity and a TM dielectric photonic crystal cavity. This nanostructure is suggested as an alternative to previous hybrid cavities, which are realized by means of metallic bow-tie antennas on dielectric cavities. Our proposed hybrid cavity works for different geometries of metallic nanoparticles including spheres, ellipsoids, or cubes. The main advantage of the proposed hybrid is that it enables nm- and sub-nm-scale gaps in a controllable way, which is out of reach in a repetitive way when the gap is defined lithographically. Such nm-scale gaps are required to get extreme small mode volumes, eventually reaching the picoscale as in standard NPoM approaches. We evidence that for a gold nanoparticle separated from a dielectric photonic cavity by a $1 \mathrm{~nm}$ gap, normalized mode volumes around $10^{-3}$ are achieved. Furthermore, the $Q$ factors exhibit high values (around $10^{3}$ ), which could even be larger by implementation of a more exhaustive design of the photonic cavity. It is the first time, to the best of our knowledge, that a feasible hybrid plasmonic-photonic cavity with high Purcell factors $\left(F_{P}=10^{5}\right)$ is proposed. In comparison with the Purcell factors obtained with feasible gaps $(d=10 \mathrm{~nm})$ for hybrid plasmonic-photonic (TE) configurations, we reach an improvement of 1 order of magnitude. This means that this hybrid configuration is able to combine the best of both configurations: high $Q$ factors due to the dielectric cavity and extremely small mode volumes (well below the diffraction limit) thanks to the metallic nanoparticle. In addition, as it was demonstrated in previous works based on bowtie antennas on dielectric cavities operating for TE polarization, changing the detuning between the cavity and the nanoparticle makes it possible to achieve different $Q$ and $V$ values. In our approach, by increasing the radius of the nanosphere from $R=$ $30 \mathrm{~nm}$ to $R=70 \mathrm{~nm}, Q$ and $V$ range from $Q_{\mathrm{HYB}}=$ $0.95 \times 10^{4}, \quad V_{\mathrm{HYB}}=2.6 \times 10^{-3}$ to $Q_{\mathrm{HYB}}=0.8 \times 10^{3}$, $V_{\mathrm{HYB}}=0.6 \times 10^{-3}$. Changing some parameters of the photonic cavity, such as the period or the nanobeam thickness, should also enable a fine tuning of $Q$ and $V$. As a next step, we plan to study our hybrid in the context of quasi-normal modes, which is becoming a powerful tool to analyze multimode open resonators [63]. This approach allows for obtaining complex volumes of different modes in a rigorous way, which should improve our analysis. Still, our results unveil a new building block in the context of hybrid plasmonic-photonic circuits [64], which should find applications in enhanced Raman scattering, harmonic generation, and molecular optomechanics even in the few-photon regime. 


\section{APPENDIX A: NUMERICAL MODEL}

In this section we present a detailed description of the model created in COMSOL to perform the simulations. Figure 8 depicts a scheme of the proposed photonic crystal cavity. It consists of a dielectric nanobeam of width $w=200 \mathrm{~nm}$ and thickness $t=250 \mathrm{~nm}$. As it can be observed, the beam is drilled with circular holes. The cavity consists of two photonic mirrors composed by a set of 10 nanoholes of period $P_{M}=165 \mathrm{~nm}$ and radius $R_{M}=43.7 \mathrm{~nm}$ at every side of the cavity. To form the cavity, an adiabatic transition was performed by changing the dimensions of the unit cells when moving toward the cavity center. In the transition section, we have reduced the period and hole size of the photonic crystal during seven holes at each side of the cavity. The period and radius for the central (defect) hole correspond to $P_{D}=138.6 \mathrm{~nm}$ and $R_{D}=36.7 \mathrm{~nm}$.

In the hybrid cavity, the NP is placed on top of the nanobeam and in the middle of the photonic crystal cavity as exhibited in Fig. 9. The gap distance (d) between the NP and the top surface of the nanobeam is $1 \mathrm{~nm}$. Figure 9(b) depicts a cut of the $3 \mathrm{D}$ geometry in the $z-x$ plane.

To perform the COMSOL simulations, the cavity was embedded in air as depicted in Fig. 10. The maximum and minimum element sizes for this region are $150 \mathrm{~nm}$ and $1.5 \mathrm{~nm}$, respectively. The air region was surrounded by a PML of thickness $500 \mathrm{~nm}$. The maximum and minimum element sizes for the NP and the beam are $45 \mathrm{~nm}$ and $30 \mathrm{~nm}$, respectively.

The electric dipole source is represented by a red point in the zoom of Fig. 11. The dipole is in the middle of the gap between the NP and the nanobeam surface, that is, $0.5 \mathrm{~nm}$ above the beam and $0.5 \mathrm{~nm}$ below the NP. The dipole moment of the electric dipole is oriented along the $z$ axis. Due to the tiny size of the gap, it was necessary to perform some convergence tests. To get accurate results, we used a finer mesh surrounding the dipole. To this aim, we created a small air sphere of

(a)

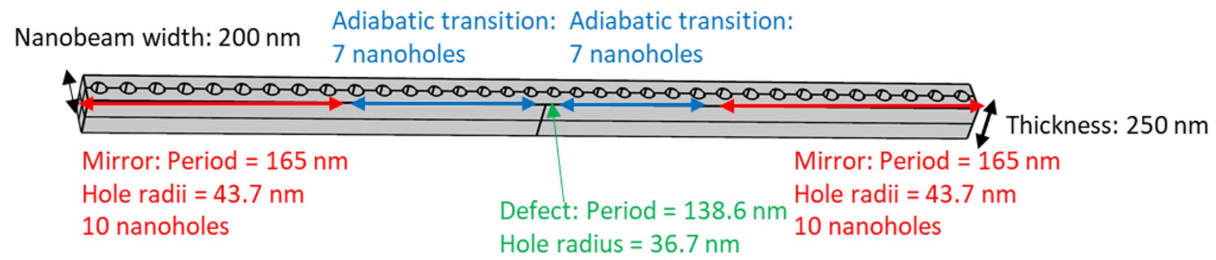

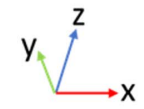

(b)

\begin{tabular}{|c|c|c|}
\hline \multicolumn{3}{|c|}{$0-0-0-0-0-0-0-0-0-0-0-0-0-0-0-0-0-0-0-0-0-0-0-0-0-0-0-0-0$} \\
\hline Mirror: Period $=165 \mathrm{~nm}$ & Adiabatic transition: Adiabatic transition: & Mirror: Period $=165 \mathrm{~nm}$ \\
\hline Hole radii $=43.7 \mathrm{~nm}$ & \begin{tabular}{l|l}
7 nanoholes & 7 nanoholes
\end{tabular} & Hole radii $=43.7 \mathrm{~nm}$ \\
\hline 10 nanoholes & $\begin{array}{l}\text { Defect: } \text { Period }=138.6 \mathrm{~nm} \\
\text { Hole radius }=36.7 \mathrm{~nm}\end{array}$ & 10 nanoholes \\
\hline
\end{tabular}

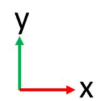

Fig. 8. Scheme of the proposed photonic crystal cavity. In (a) and (b), 3D and 2D ( $x-y$ plane) views are shown.

(a)
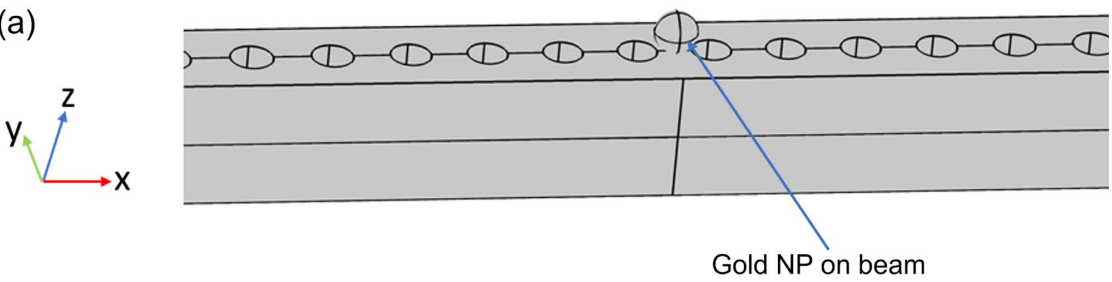

(b)
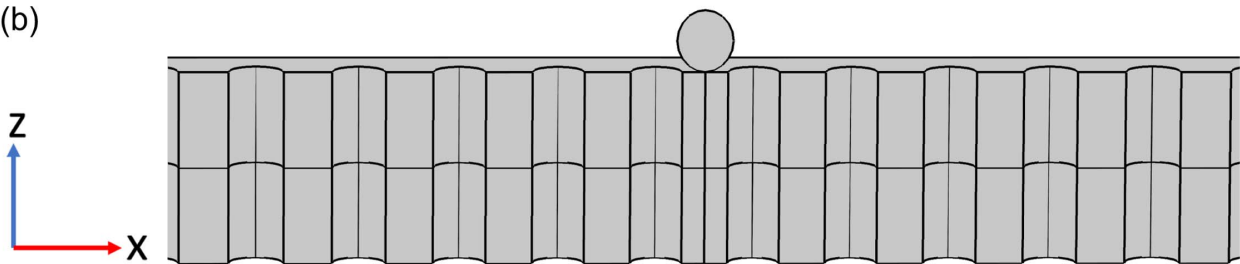

Fig. 9. Scheme of the hybrid cavity: gold NP—in this case, a nanosphere—on a photonic crystal cavity. (a) and (b) A 3D view and a 2D cut ( $z-x$ plane) of the geometry, respectively. 


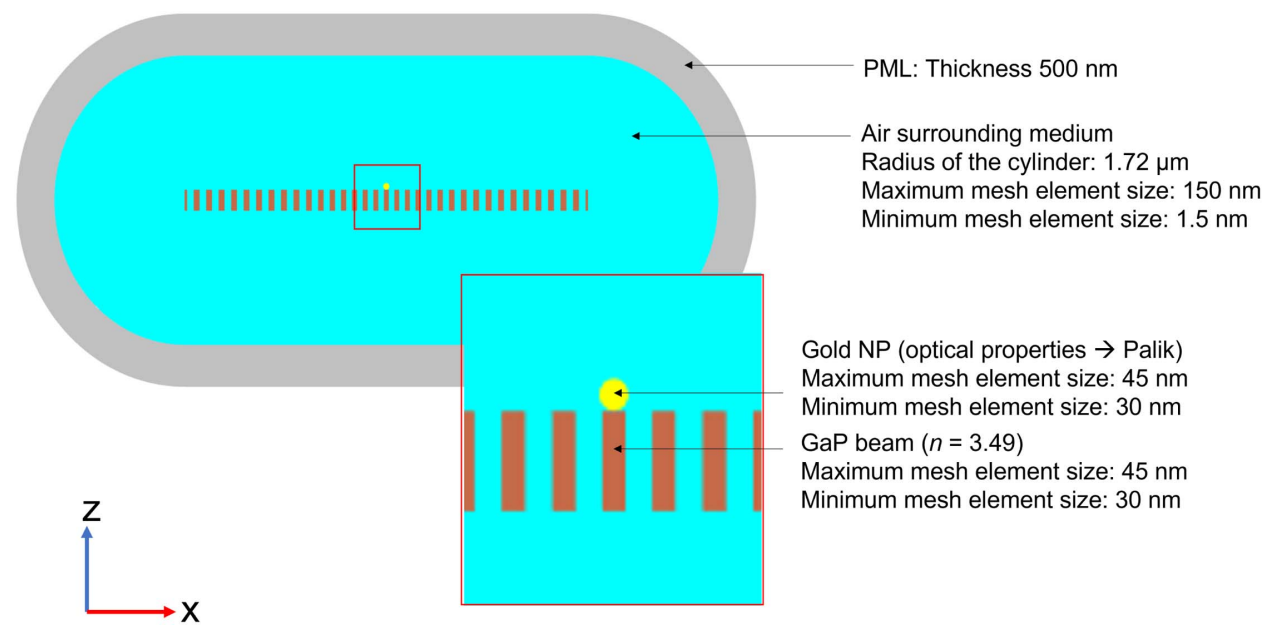

Fig. 10. $z-x$ cut of the geometry simulated with COMSOL, showing the hybrid cavity at the center. The structure is embedded in an air cylindrical region. This air region is surrounded by a PML.

(a)

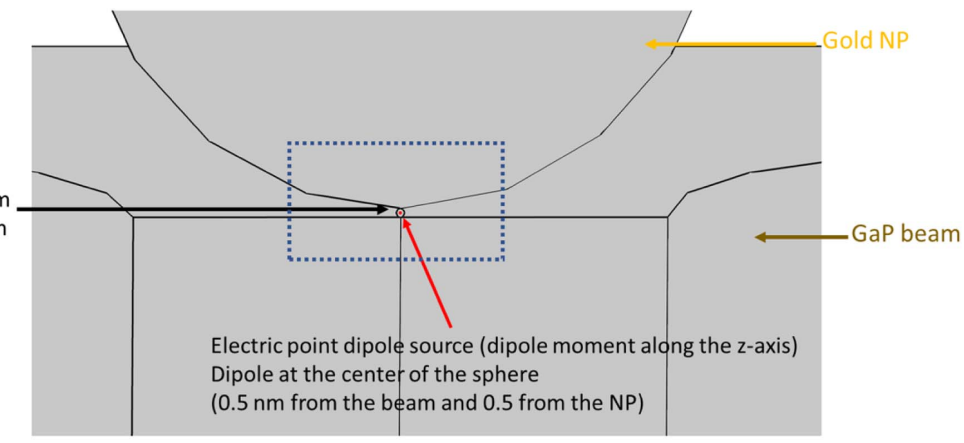

(b)

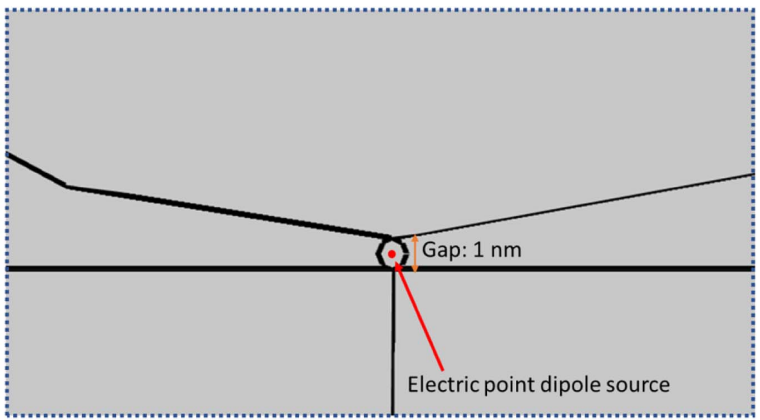

Fig. 11. Detail of the $z-x$ cut of the geometry simulated with COMSOL. (a) Graphical representation of the gold NP, the nanobeam boundary, and the electric dipole source. In (b) we show a zoom of the gap between the NP and the cavity, where the dipolar source is placed. The small air sphere surrounding the dipole- used to achieve the convergence of the results—is also plotted.

diameter equal to the size of the gap. The maximum and minimum element meshes for this sphere were $1.2 \mathrm{~nm}$ and $0.6 \mathrm{~nm}$, respectively.

To calculate the radiative part of the LDOS, it is necessary to create an additional smaller cylinder in the center of the larger cylinder and made of air. The radius of this cylinder is $1.11 \mu \mathrm{m}$ (see Fig. 12). The scattered power $\left(P_{\text {rad }}\right)$ is calculated at the boundaries of the smaller cylinder, while the total power loss density is obtained by means of the volume integration of the losses in the metallic nanoparticle.

It is worth remarking that we performed two different kinds of simulations with two distinct solvers. To obtain the nearfield maps, we used the eigenfrequency solver. In that case, there is no illumination source. However, to retrieve the 
(a)
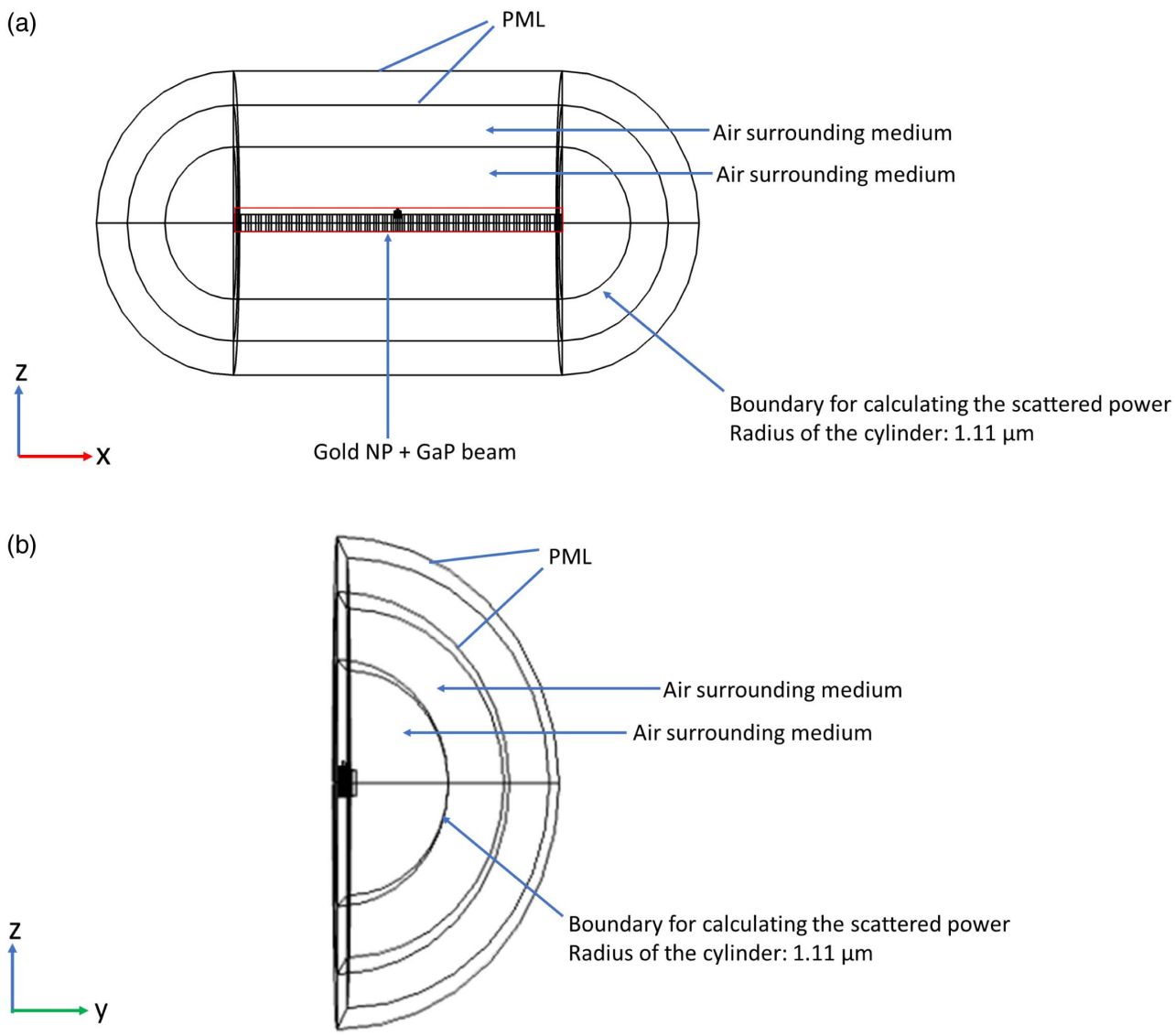

(c)

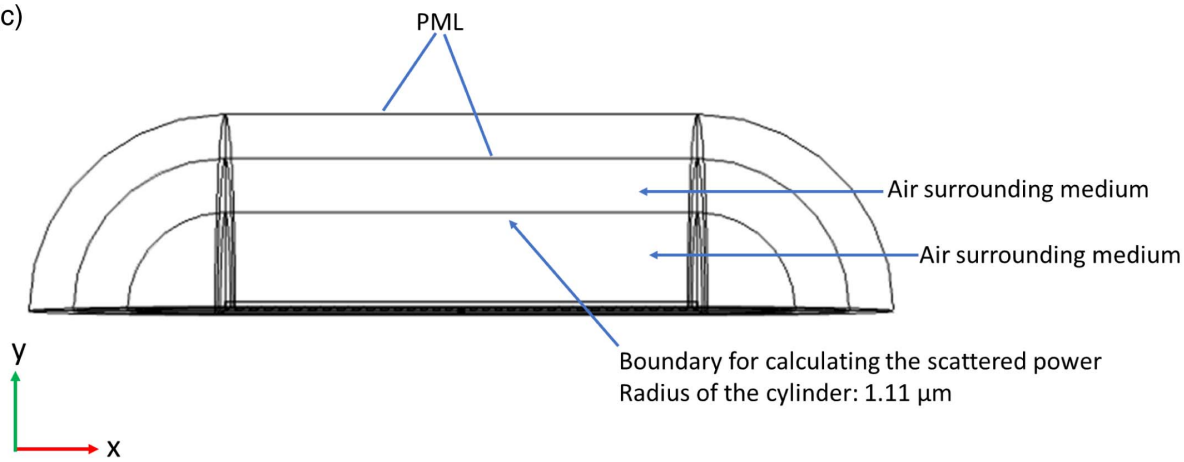

Fig. 12. Scheme of the geometry used in COMSOL to calculate the radiative power. (a) $z-x$ plane. (b) $z-y$ plane. (c) $x-y$ plane.

(a)

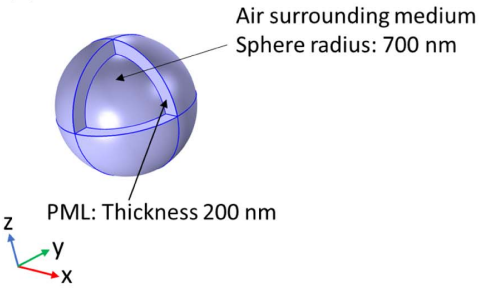

(b)

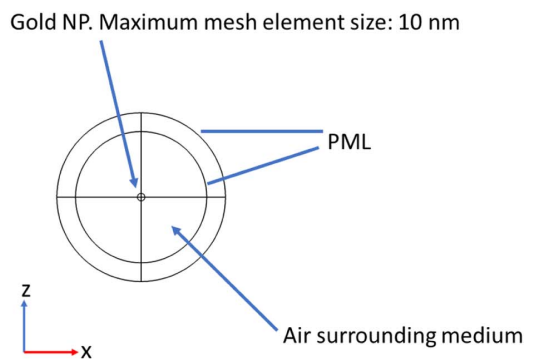

Fig. 13. Scheme of the model used to perform the simulations corresponding to the absorption, scattering, and extinction cross sections of bare gold NPs. In (a), the PML and the air medium surrounding the NP can be observed. In (b), we have represented a 2D cut of the 3D view in (a), where the NP is also visible. 
LDOS, the simulations were performed with the frequencydomain solver. For these calculations, a dipole source was included. Although the simulations are different, we have used the same geometry and mesh to avoid possible numerical errors. The only near-field map that was attained by using the frequency-domain solver, with a plane wave illumination, is that corresponding to the isolated nanosphere [Fig. 2(b)]. The reason is that with this calculation, from the scattered field distribution, we are also able to identify the excited mode (dipolar electric), and the computation is faster than calculating the eigenfrequency values. To obtain the absorption, scattering, and extinction cross sections of the bare NPs, and the near-field map in Fig. 2(b) (corresponding to a nanosphere), a simpler model can be used with COMSOL. The bare NP is located at the center of a larger sphere of diameter $700 \mathrm{~nm}$ and made of air. This air region is surrounded by a PML of thickness $200 \mathrm{~nm}$; see Fig. 13. The structure is illuminated with a plane wave linearly polarized along the $x$ axis and propagating along the $z$ axis. To ensure numerical convergence of the results, the mesh of the NP was sufficiently fine, being smaller than $10 \mathrm{~nm}$. In particular, the maximum mesh element size was $10 \mathrm{~nm}$, and the minimum one was $0.36 \mathrm{~nm}$.

\section{APPENDIX B: CONVERGENCE TESTS}

In this section we present some tests to demonstrate the convergence and accuracy of our simulations. As a first check, we compare the radiative power emitted by the dipole in the presence of the cavity and the NP when the radius of the air sphere surrounding the dipole is changed from $R=0.2 \mathrm{~nm}$ to $R=0.4 \mathrm{~nm}$. It is important to notice that we present the radiative LDOS without normalizing the results to the radiative power emitted by the dipole in vacuum, as our purpose is to prove the convergence of the power flow. The results are shown in Fig. 14(a). We can see how the same values for the radiative part of the LDOS are attained independently of the radius of the sphere surrounding the dipole. As a second check, we calculate the LDOS by means of two different methods.

(1) LDOS as the sum of the radiative and nonradiative power, normalized to the radiative power of the dipole in vacuum [Eq. (2)].

(2) LDOS calculated using the definition of Green's function [4] as the negative real part of the scattered electric field by

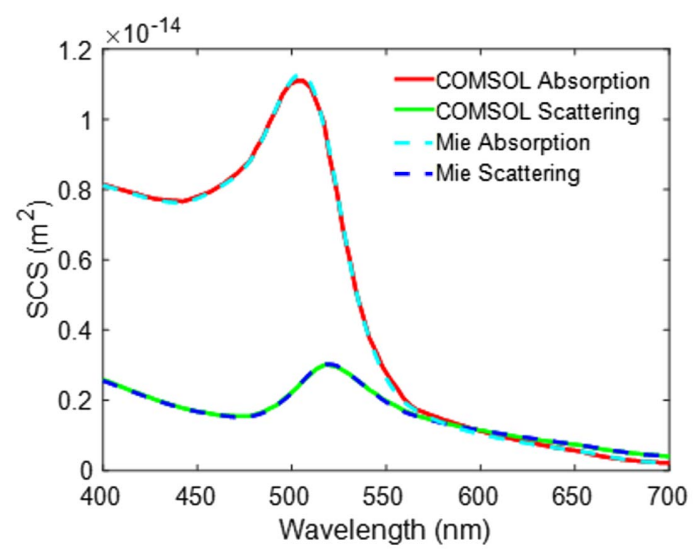

Fig. 15. Absorption and scattering cross sections' spectra for a nanosphere of radius $R=40 \mathrm{~nm}$ obtained numerically, with COMSOL, and analytically, by means of the Mie theory.

the dipole (oriented in the $z$ direction) at the position of the point source emitter.

To examine the convergence of the calculations based on plane wave illumination, in Fig. 15 we compare the absorption and scattering cross sections' spectra obtained numerically, with COMSOL, and analytically, by means of the Mie theory. A clear agreement between both of them is seen.

\section{APPENDIX C: CURVE FITTINGS}

In this section we provide a deeper explanation about the reasons why either Fano or Lorentzian curves were used for the fittings in the main text, depending on the considered system (bare cavity, bare nanoparticle, or hybrid system, constituted by a metallic NP coupled to a dielectric nanobeam cavity). In addition, we clarify how the $Q$ factor can be retrieved from these fittings. For the case of the bare cavity, the LDOS is fitted by a Lorentzian curve. This fitting is chosen because only the cavity mode is excited, which in our work corresponds to the fundamental TM mode of the cavity. In that case, as expected, the shape of the curve is symmetric [see Fig. 2(c)]. However, for the hybrid, the line shape is no longer Lorentzian but slightly asymmetric following a Fano line shape. Such a Fano line shape is characteristic of interference between a narrow resonance and
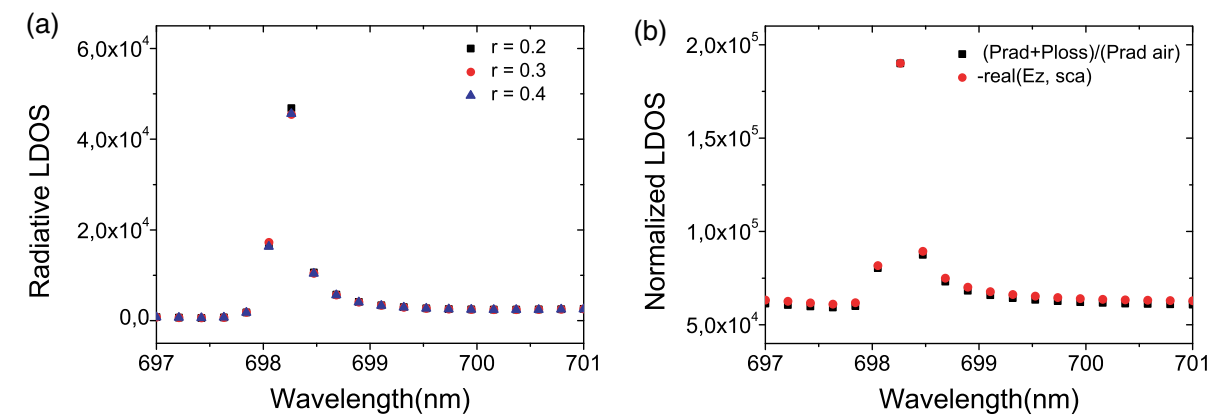

Fig. 14. (a) Radiative LDOS for different radii of the sphere surrounding the dipole $(r=0.2-0.4 \mathrm{~nm})$ for a nanosphere-based hybrid cavity (radius of the nanosphere $R=40 \mathrm{~nm}$ ). (b) Normalized LDOS calculated by the methods (1) and (2) described above for a nanosphere-based hybrid cavity (radius of the nanosphere $R=40 \mathrm{~nm}$ ). 
a broader background, and it has been predicted by several groups to occur in the hybrid cavity-antenna system LDOS [10,13,65-67]. The reason is that for the hybrid system there is no longer a single mode but the hybridization of the excited mode in the metallic NP with the mode of the dielectric cavity. In our system this corresponds to the hybridization of the fundamental TM cavity mode with the plasmonic mode in the gold nanoparticle; see Fig. 3(a). Although the main mode excited in the bare nanoparticles is the dipolar electric one, due to the illuminating dipole source in close proximity to the NP $(0.5 \mathrm{~nm})$, higher-order modes can also contribute to the resonance peak. For that reason, the fitting of the bare nanoparticle is performed by means of a Fano curve instead of a Lorentzian one; see Fig. 2(a). In the case that only a single mode was excited in the metallic NP, the shape of the curve would be symmetric, and the fitting would be performed by means of a Lorentzian shape. It is necessary to note that although higher-order modes are excited in the metallic nanoparticles (especially for the nanocube and nanoellipsoid), the main contribution corresponds to the dipolar electric mode. For that reason, it is reasonable to perform a Fano fitting for the nanoparticle-cavity hybrid system.
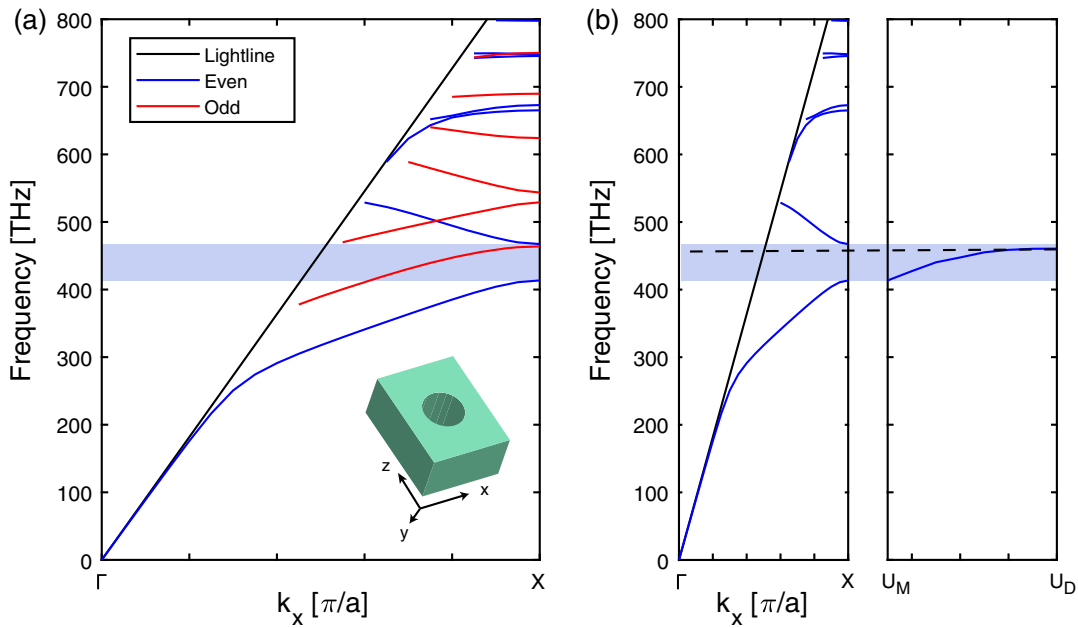

Fig. 16. (a) Photonic band diagram of the mirror unit cell for the TM modes with even and odd $z$ symmetries. (b) (Left) Close view of the even symmetric modes and (right) evolution of the confined band at the $\mathrm{X}$ symmetry point from the mirror unit cell to the defect unit cell.
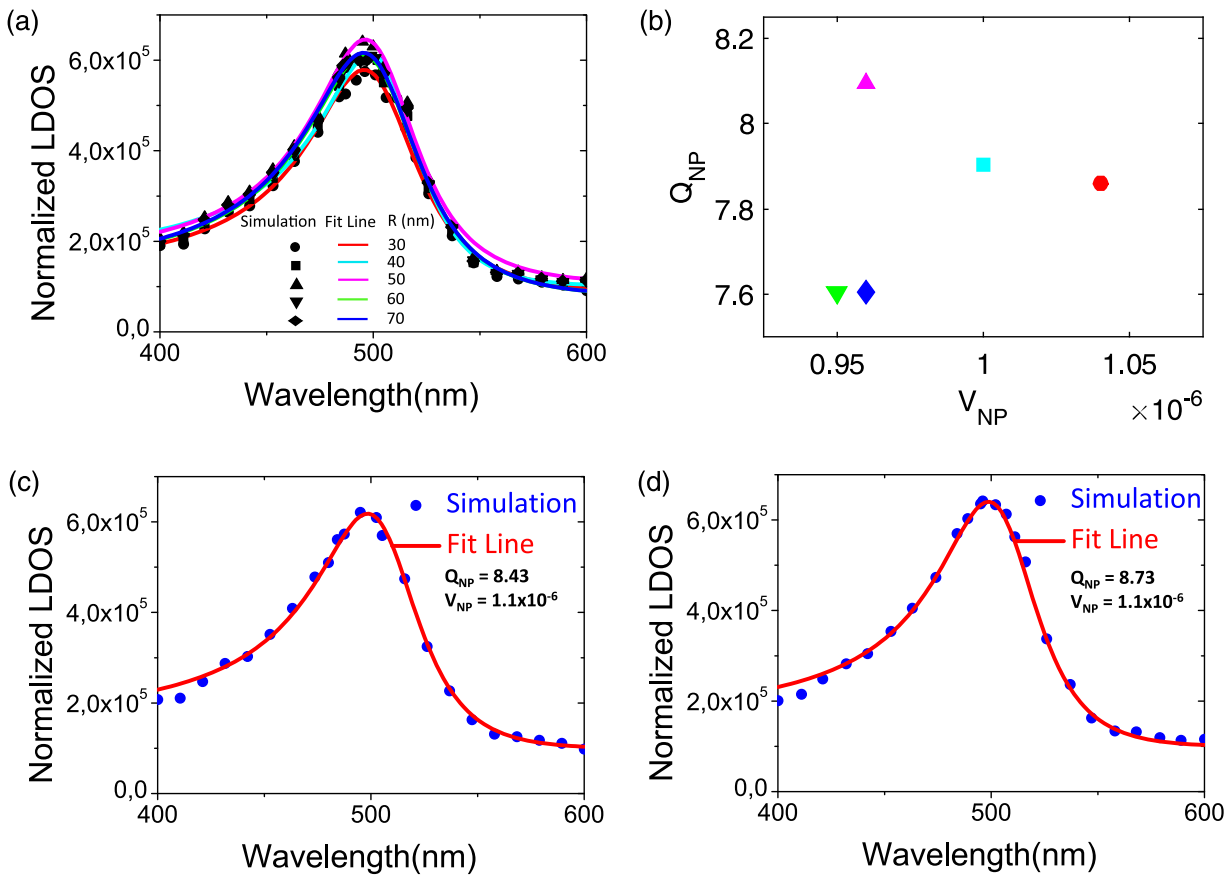

Fig. 17. (a) Normalized LDOS and (b) $Q$ factor and $V$ for the bare nanosphere as a function of the radius $(R$ ranging from 30 to $70 \mathrm{~nm})$. The normalized LDOS for the nanocube $(l=75 \mathrm{~nm})$ and nanoellipsoid $\left(w_{e}=40 \mathrm{~nm}\right.$ and $\left.l_{e}=70 \mathrm{~nm}\right)$ is represented in (c) and (d), respectively. As insets, the $Q$ factor and $V$ values are provided. 
The equations for the Lorentzian and Fano fittings correspond to Eqs. (C1) and (C2), respectively:

$$
y=y_{0}+\frac{2 A}{\pi} \frac{w}{\left(x-x_{c}\right)^{2}+w^{2}},
$$

where $y_{0}$ is the offset, $A$ is a multiplicative constant, $w$ is the full width at half-maximum, and $x_{c}$ corresponds to the resonance wavelength, and

$$
y=y_{0}+A \frac{\left(q w+x-x_{c}\right)^{2}}{\left(x-x_{c}\right)^{2}+w^{2}},
$$

where $q$ is the Fano parameter, $y_{0}$ is the offset, $A$ is a multiplicative constant, $w$ is the full width at half-maximum, and $x_{c}$ is the resonance wavelength.

From the fitting of the simulation points to the Lorentzian or Fano curves, it is possible to retrieve the $Q$-factor values by means of the parameters $x_{c}$ and $w$. In fact, $Q$ can be retrieved as follows:

$$
Q=\frac{x_{c}}{2 w}
$$

\section{APPENDIX D: PHOTONIC BAND DIAGRAM}

Figure 16 represents the photonic band diagram of the mirror unit cell for the TM field modes. Even and odd $z$ symmetries for the mirror cell are considered. The evolution of the confined band from the mirror (sides) to the defect cell (center) is also plotted. A TM bandgap for guided modes appears around $440 \mathrm{THz}$ (corresponding to a wavelength around $681 \mathrm{~nm}$ ). The bandgap width is $54 \mathrm{THz}$. As it is inferred from the evolution to the defect unit cell, its geometrical parameters were chosen to exhibit a band in the middle of the bandgap of the photonic crystal.
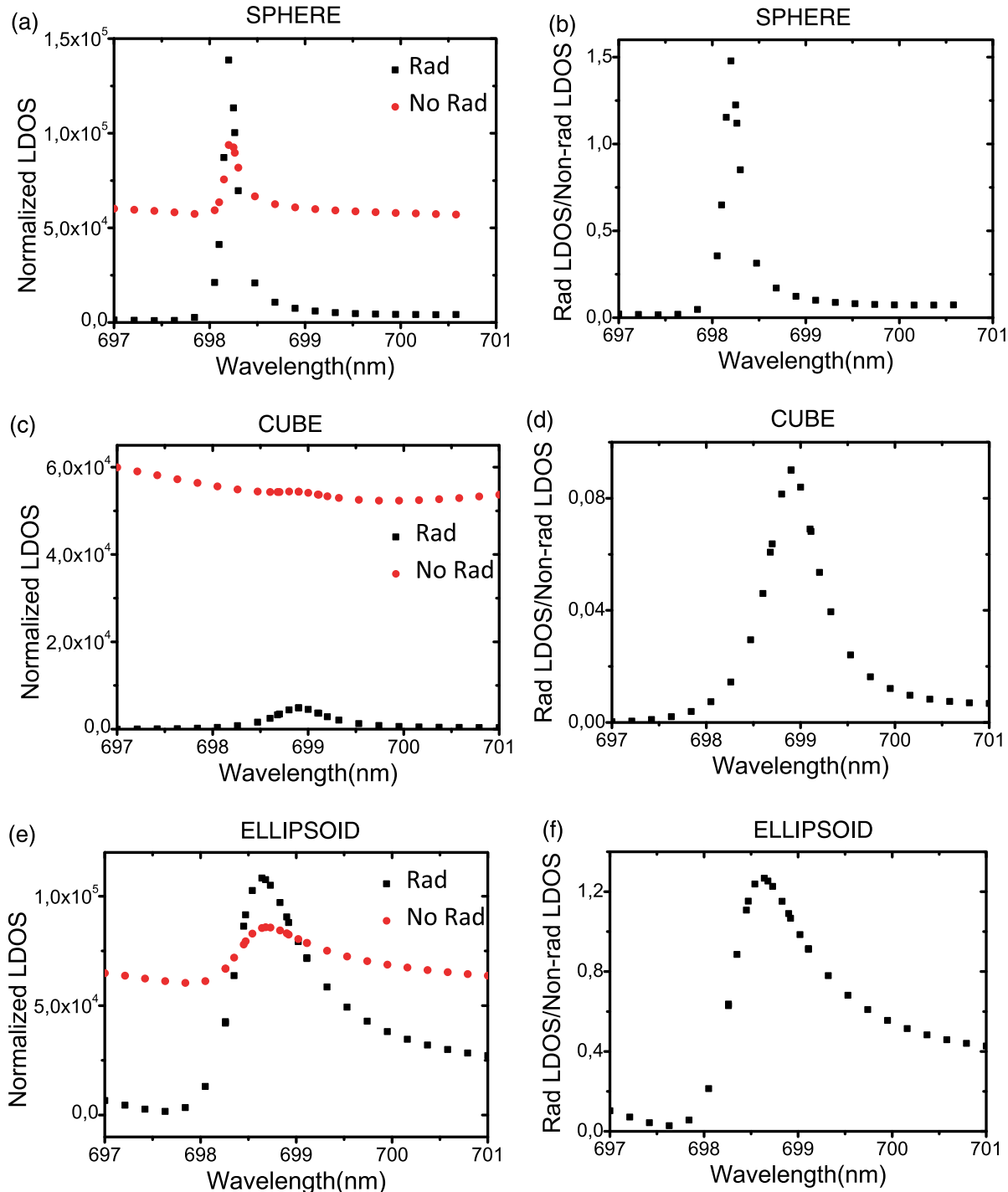

Fig. 18. Radiative and nonradiative contributions to the normalized LDOS for the (a) nanosphere-based $(R=40 \mathrm{~nm})$, (c) nanocube-based $(l=75 \mathrm{~nm})$, and (e) nanoellipsoid-based hybrid cavities $\left(w_{e}=40 \mathrm{~nm}\right.$ and $\left.l_{e}=70 \mathrm{~nm}\right) . \mathrm{In}(\mathrm{b}),(\mathrm{d})$, and (f) the ratio radiative/nonradiative LDOS is represented for the same configurations as in (a), (c), and (e). 


\section{APPENDIX E: ISOLATED NANOSPHERES}

Figure 17 shows the LDOS, $Q$ factor, and $V$ for the isolated nanoparticles. Figure 17(a) shows the normalized LDOS for the nanospheres with radius varying from $R=30$ to $70 \mathrm{~nm}$. The corresponding $Q$ factors and $V$ values are shown in Fig. 17(b). Figures 17(c) and 17(d) plot the normalized LDOS for the nanocube and nanoellipsoid, respectively. As an inset, the values of the $Q$ factor and $V$ are provided for each one of the geometries. The normalized LDOS is obtained by considering the illumination of an electric dipole placed $0.5 \mathrm{~nm}$ below the nanoparticle, whose dipole moment is oriented along the $z$ axis. The parameters of the nanoparticle, $Q_{\mathrm{NP}}$ and $V_{\mathrm{NP}}$, are retrieved through the fitting of the LDOS to a Fano line shape.

\section{APPENDIX F: RADIATIVE AND NONRADIATIVE POWER CONTRIBUTIONS}

Figures 18(a), 18(c), and 18(e) represent the contributions of the radiative and nonradiative power to the normalized LDOS for the different nanoparticle-based hybrid cavity configurations (nanosphere, nanocube, and nanoellipsoid). The radius of the nanosphere corresponds to $R=40 \mathrm{~nm}$. It is observed
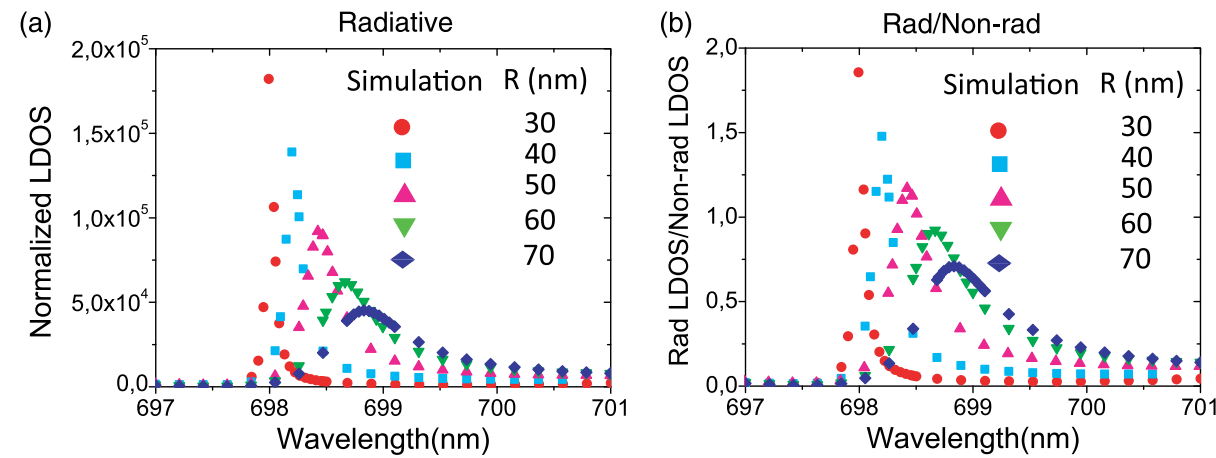

Fig. 19. Radiative contribution to the (a) normalized LDOS and (b) ratio of radiative/nonradiative LDOS for different radii of the nanospherebased hybrid cavity $(R=[30-70] \mathrm{nm})$.

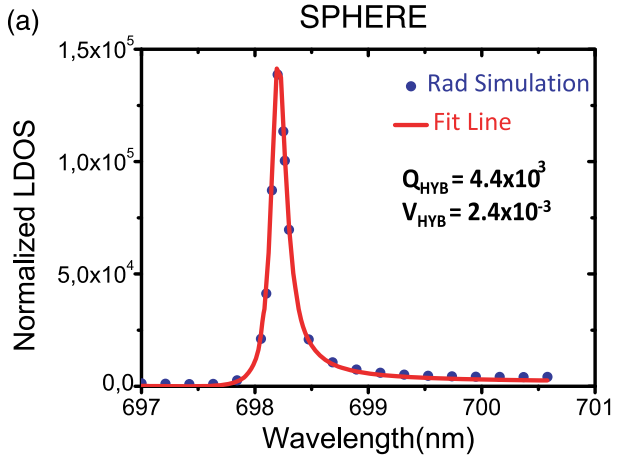

(c)

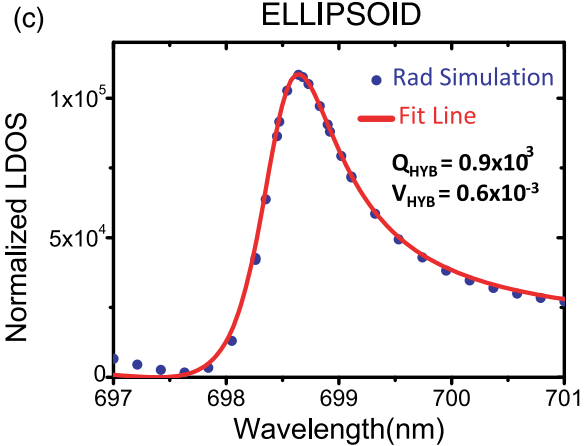

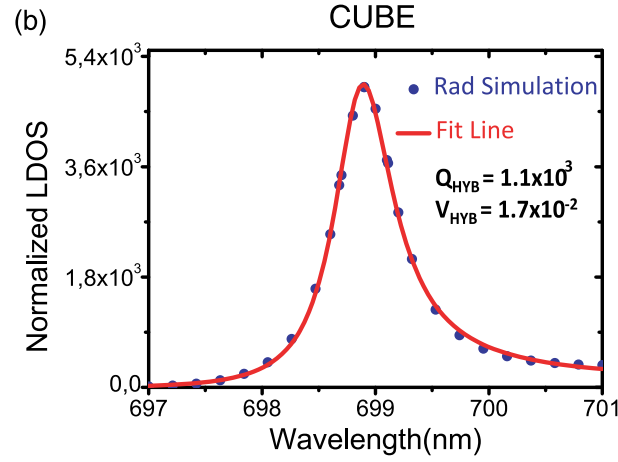

(d)

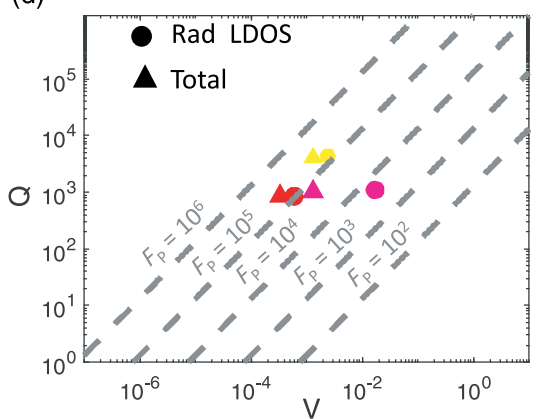

Fig. 20. Normalized LDOS, $Q$ factor, and $V$ for (a) nanosphere-based (radius $R=40 \mathrm{~nm}$ ), (b) nanocube-based (side length of the nanocube $l=75 \mathrm{~nm}$ ), and (c) nanoellipsoid-based hybrid cavity (the width and length of the ellipsoid are $w_{e}=40 \mathrm{~nm}$ and $l_{e}=70 \mathrm{~nm}$, respectively). Only the radiative contribution has been considered. (d) $Q$ and $V$ for the hybrids considering the total LDOS (radiative + nonradiative contributions) (triangles) or only the radiative LDOS (circles) for different geometries of nanoparticles (sphere, yellow; ellipsoid, red; and cube, magenta). Diagonal dashed lines represent constant Purcell factor with value $F_{P}$ as labeled. 
that, at the resonance wavelength, for the nanosphere, the radiative power takes larger values than the nonradiative power, obtaining a ratio of radiative/nonradiative LDOS of 1.5 [see Fig. 18(b)], which is the largest of the three studied geometries. For the case of the ellipsoid, the radiative contribution is also larger than the nonradiative one. In fact, the ratio of radiative/ nonradiative LDOS takes a value of 1.3 at the resonance wavelength [Fig. 18(f)]. For the nanocube, the radiative power is small compared to the nonradiative power. The radiative efficiency for this shape is quite low [the ratio of radiative/nonradiative LDOS is 0.09; Fig. 18(d)]. For that reason, spheres and ellipsoids are more promising structures, as they show high $Q$ factor and small $V$ values, but at the same time preventing of high emission quenching. However, it is worth remarking that even in the worst case (cube), the hybrid mode can be experimentally excited. The reasons why the radiative efficiency is lower for the cube than for the sphere or ellipsoid are provided in Section 4.
As mentioned above, the sphere is the geometry that provides the largest radiative efficiency. To analyze more deeply this behavior, in Fig. 19 we represent the evolution of the radiative contribution to LDOS and the ratio between the radiative and nonradiative power as a function of the nanosphere radius in a nanosphere-based hybrid cavity. The chosen sizes are the same as in the manuscript $(R=30-70 \mathrm{~nm})$. It is observed that the radiative emitted radiation as well as the ratio radiative to nonradiative emission increases when the radius of the nanosphere decreases. In particular, for $R=30 \mathrm{~nm}$, the radiative/ nonradiative LDOS reaches a value of 2 . However, for $R=$ $70 \mathrm{~nm}$, the ratio is 0.7 . This effect can be understood attending to the mode excitation in the metal NP. As the dipolar character becomes predominant (smaller radii), the hybridization between the modes of the cavity and the NP is larger, increasing the radiative power density with respect to the power loss density. In addition, it should be noted that a change of radius for the nanosphere also modifies the width of the resonance and (a)

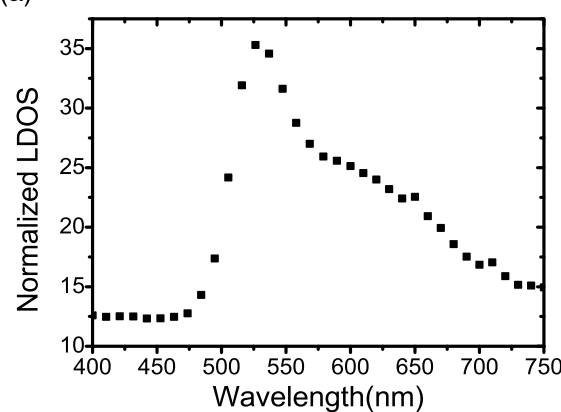

(c)

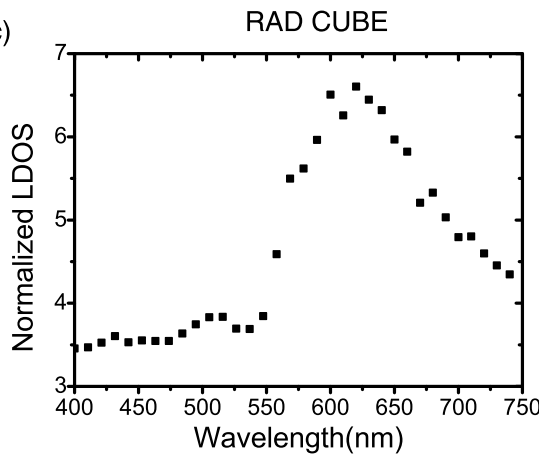

(e)

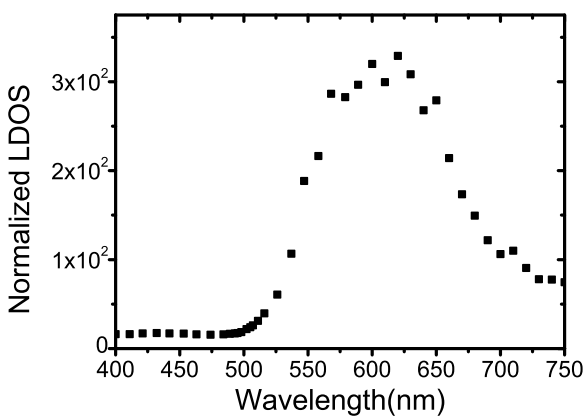

(b)

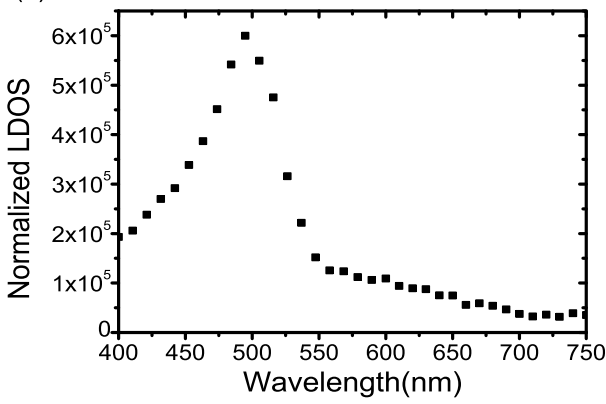

(d)

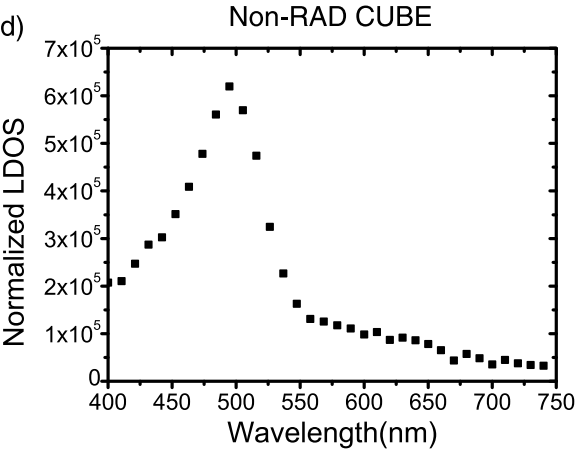

(f)

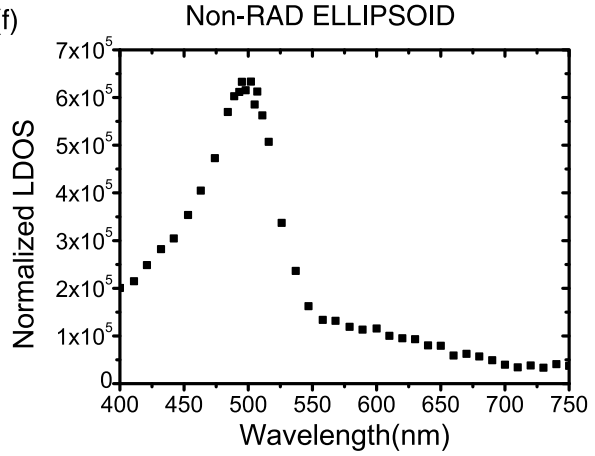

Fig. 21. Radiative contribution to the normalized LDOS for the (a) nanosphere $(R=40 \mathrm{~nm})$, (c) nanocube $(l=75 \mathrm{~nm})$, and (e) nanoellipsoid $\left(w_{e}=40 \mathrm{~nm}\right.$ and $\left.l_{e}=70 \mathrm{~nm}\right)$. (b), (d), and (f) Nonradiative contribution to LDOS for the same configurations as in (a), (c), and (e). 
the mode volume of the system. This is due to the radius of curvature changing the extension of the light localization in the coupled mode. This leads to two different effects. On the one hand, as the size of the nanosphere increases, the metallic (and therefore lossy) area between the nanoparticle and the dipole increases. This is responsible for an increase in the nonradiative power and a decrease in the ratio $\mathrm{rad} /$ nonradiative LDOS. On the other hand, when the radius of the nanosphere increases, the light is more confined between the NP and the cavity, decreasing $V$.

In previous works, the enhancement of the emission of quantum emitters embedded in different nanostructures was analyzed. In particular, gold dimers with small gap distances between their components were proposed to generate large concentrations of electromagnetic radiation and small mode volumes. The enhanced emission of an emitter placed in the gap was redirected in a certain direction by means of silicon antennas $[68,69]$. It is interesting to compare the radiation efficiency (ratio of the radiative power with respect to the total power) of our hybrid system with those other configurations. In Ref. [68], a gold bow-tie antenna provided strong electromagnetic energy concentration at the emitter position, and three silicon nanorods were responsible for guiding the radiation in a certain direction. A radiation efficiency of 0.5 was reported. In Ref. [69], the role of the bow-tie antennas is played by a gold nanorod dimer, and the silicon nanorods are substituted by a silicon cylinder. In that article, authors claimed a radiation efficiency of 0.7. For the cases of nanosphere and nanoellipsoids, we get a radiation efficiency of $0.6-0.7$, which is comparable to or even better than previous designs. This demonstrates the potential of these hybrid configurations based on the coupling of NP with photonic crystals in quantum optics. Since for many practical applications the radiative part is the most important contribution, in Figs. 20(a)-20(c) we have represented
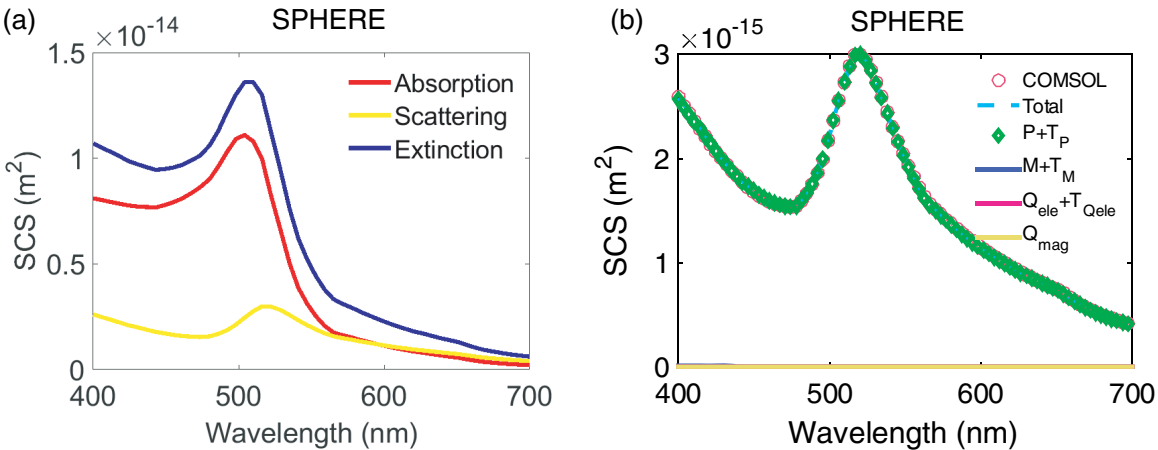

(c)
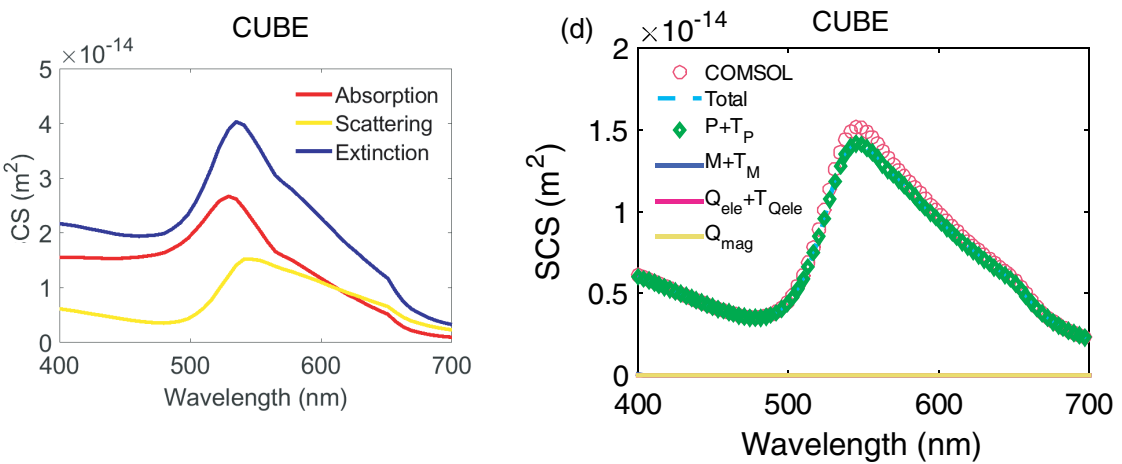

(e)
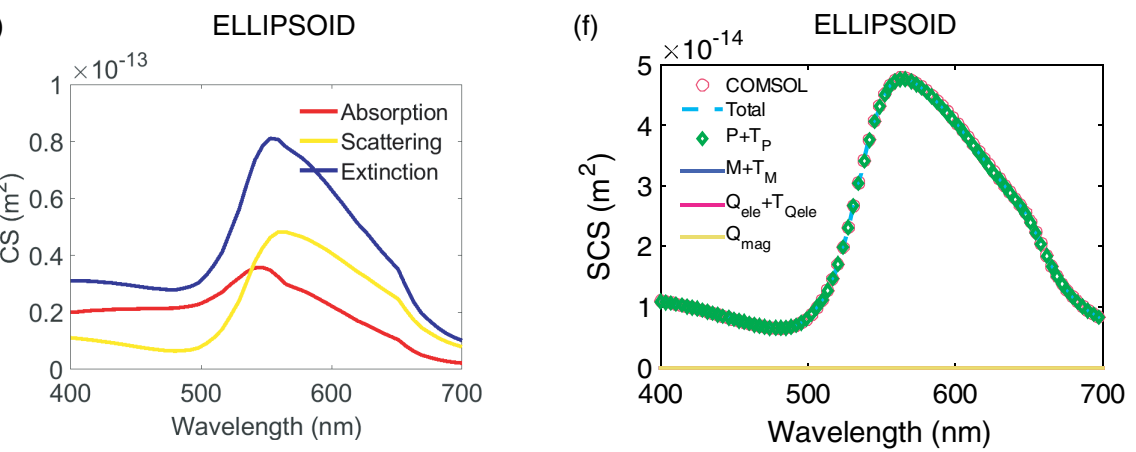

Fig. 22. Absorption, scattering, and extinction cross sections (CS) for the (a) nanosphere $(R=40 \mathrm{~nm})$, (c) nanocube $(l=75 \mathrm{~nm})$, and (e) nanoellipsoid ( $w_{e}=40 \mathrm{~nm}$ and $l_{e}=70 \mathrm{~nm}$ ). (b), (d), and (f) Multipolar decomposition corresponding to the scattering cross sections (SCS) for the same configurations as in (a), (c), and (e). The NPs are illuminated by a plane wave linearly polarized along the $z$ axis and propagating along the $x$ axis (see Fig. 1 for axis orientation). 
the LDOS of the hybrid systems for the nanosphere $(R=$ $40 \mathrm{~nm}$ ), nanocube, and nanoellipsoid, but examining only the radiative part (radiative LDOS). The LDOS is obtained by means of the illumination of an electric dipole located $0.5 \mathrm{~nm}$ above the cavity, whose dipole moment is oriented along the $z$ axis. From the fitting of the curve to a Fano function, the parameters of the hybrid $\left(Q_{\mathrm{HYB}}\right.$ and $\left.V_{\mathrm{HYB}}\right)$ are retrieved. By means of the comparison of the results, taking into account the radiative and nonradiative contribution or only the radiative one [triangles and circles in Fig. 20(d), respectively], we can conclude that similar $Q_{\mathrm{HYB}}$ and $V_{\mathrm{HYB}}$ values are obtained. This suggests that our proposed hybrid systems are able to improve the $Q$ and $V$ values with respect to the bare components (nanoparticles and cavities) when only the radiative part is considered. The largest difference is attained for the mode volume of the hybrid nanocube-cavity. This is due to the large contribution of the nonradiative part for this shape.

The radiative and nonradiative contributions to the LDOS for the bare nanoparticles are shown in Fig. 21. We have represented these magnitudes for the nanosphere $(R=40 \mathrm{~nm})$, the nanocube, and the nanoellipsoid. We can observe that for all the geometries, the dominant contribution is the nonradiative part, as it was expected for a metallic NP illuminated by a dipole in its proximity (the distance from the dipole to the metallic NP $d=0.5 \mathrm{~nm}$ ). The fact that most of the emission is nonradiative is due to the dipole illumination and the short distance between the dipole and the nanoscatterer. In fact, in Fig. 22 we can observe that, although the absorption plays an important role, its contribution to the extinction cross section is not so large as the contribution of the nonradiative part to the total LDOS for the dipole illumination. It is important to remark that for the cross-section calculations, the nanoparticles were illuminated with a plane wave linearly polarized along the $z$ axis and propagating along the $x$ axis (see Fig. 1 for axis orientation).

Through the comparison of the bare nanoparticles with the hybrid systems, it is clear that the hybridization is responsible for the increase of the radiative part with respect to the nonradiative one.

In Figs. 22(a), 22(c), and 22(e), we only observe one resonance in the spectrum. To understand which modes are responsible for the resonances, we have performed a multipolar decomposition for the nanosphere $(R=40 \mathrm{~nm})$, nanocube, and nanoellipsoid. The results are found in Figs. 22(b), 22(d), and 22(f). It is observed that for all the analyzed geometries, the resonance has a dipolar electric character. However, as it was just pointed out, contributions of higher-multipolar orders may appear when the NPs are illuminated by an electric dipole, instead of a plane wave (as it is done for attaining the multipolar decomposition). The excitation of the higher-multipolar orders is responsible for the discrepancies observed between the $\mathrm{CHO}$ model and the numerical simulations and for the differences in the ratios of scattering/absorption and radiative/nonradiative LDOS.

Funding. Horizon 2020 Framework Programme (829067 THOR); Generalitat Valenciana (PPC/2018/002, PROMETEO/2019/123); Ministerio de Ciencia, Innovación y Universidades (PGC2018-094490-B, PRX18/00126); Alexander von Humboldt-Stiftung.

Acknowledgment. We thank C. Galland for useful discussions. A. B. acknowledges financial support by the Alexander von Humboldt Foundation. A. M. acknowledges support from the Spanish Ministerio de Ciencia, Innovación y Universidades, and the Generalitat Valenciana.

Disclosures. The authors declare no conflicts of interest.

Data Availability. Data underlying the results presented in this paper are not publicly available at this time but may be obtained from the authors upon reasonable request.

\section{REFERENCES}

1. E. M. Purcell, "Spontaneous emission probabilities at radio frequencies," Phys. Rev. 69, 681 (1946).

2. A. F. Koenderink, "On the use of Purcell factors for plasmon antennas," Opt. Lett. 35, 4208-4210 (2010).

3. X. Zambrana-Puyalto and N. Bonod, "Purcell factor of spherical Mie resonators," Phys. Rev. B 91, 195422 (2015).

4. L. Novotny and B. Hecht, Principles of Nano-Optics, 2nd ed. (Cambridge University, 2012).

5. R. Sprik, B. A. van Tiggelen, and A. Lagendijk, "Optical emission in periodic dielectrics," Europhys. Lett. 35, 265-270 (1996).

6. T. Asano, Y. Ochi, Y. Takahashi, K. Kishimoto, and S. Noda, "Photonic crystal nanocavity with a $Q$ factor exceeding eleven million," Opt. Express 25, 1769-1777 (2017).

7. K. J. Vahala, "Optical microcavities," Nature 424, 839-846 (2003).

8. J. A. Schuller, E. S. Barnard, W. Cai, Y. C. Jun, J. S. White, and M. L. Brongersma, "Plasmonics for extreme light concentration and manipulation," Nat. Mater. 9, 193-204 (2010).

9. F. Benz, M. K. Schmidt, A. Dreismann, R. Chikkaraddy, Y. Zhang, A. Demetriadou, C. Carnegie, H. Ohadi, B. de Nijs, R. Esteban, J. Aizpurua, and J. J. Baumberg, "Single-molecule optomechanics in "picocavities'," Science 354, 726-729 (2016).

10. H. M. Doeleman, E. Verhagen, and A. F. Koenderink, "Antenna-cavity hybrids: matching polar opposites for Purcell enhancements at any linewidth," ACS Photon. 3, 1943-1951 (2016).

11. G. M. Akselrod, C. Argyropoulos, T. B. Hoang, C. Ciracì, C. Fang, J. Huang, D. R. Smith, and M. H. Mikkelsen, "Probing the mechanisms of large Purcell enhancement in plasmonic nanoantennas," Nat. Photonics 8, 835-840 (2014).

12. I. Mukherjee, G. Hajisalem, and R. Gordon, "One-step integration of metal nanoparticle in photonic crystal nanobeam cavity," Opt. Express 19, 22462-22469 (2011).

13. M. Kamandar Dezfouli, R. Gordon, and S. Hughes, "Modal theory of modified spontaneous emission of a quantum emitter in a hybrid plasmonic photonic-crystal cavity system," Phys. Rev. A 95, 013846 (2017).

14. I. Mukherjee and R. Gordon, "Analysis of hybrid plasmonic-photonic crystal structures using perturbation theory," Opt. Express 20, 1699217000 (2012).

15. M. K. Dezfouli, R. Gordon, and S. Hughes, "Molecular optomechanics in the anharmonic cavity QED regime using hybrid metal-dielectic cavity modes," ACS Photon. 6, 1400-1408 (2019).

16. I. M. Palstra, H. M. Doeleman, and A. F. Koenderink, "Hybrid cavityantenna systems for quantumoptics outside the cryostat?" Nanophotonics 8, 1513-1531 (2019).

17. H. M. Doeleman, C. D. Dieleman, C. Mennes, B. Ehler, and A. F. Koenderink, "Observation of cooperative Purcell enhancements in antenna-cavity hybrids," ACS Nano 14, 12027-12036 (2020).

18. H. Zhang, Y.-C. Liu, C. Wang, N. Zhang, and C. Lu, "Hybrid photonicplasmonic nano-cavity with ultra-high Q/V," Opt. Lett. 45, 4797 (2020). 
19. E. Arbabi, S. M. Kamali, S. Arnold, and L. L. Goddard, "Hybrid whispering gallery mode/plasmonic chain ring resonators for biosensing," Appl. Phys. Lett. 105, 231107 (2014).

20. Y. Hong, W. Ahn, S. V. Boriskina, X. Zhao, and B. M. Reinhard, "Directed assembly of optoplasmonic hybrid materials with tunable photonic-plasmonic properties," J. Phys. Chem. Lett. 6, 2056-2064 (2015).

21. C. Klusmann, R. N. S. Suryadharma, J. Oppermann, C. Rockstuhl, and $\mathrm{H}$. Kalt, "Hybridizing whispering gallery modes and plasmonic resonances in a photonic metadevice for biosensing applications," J. Opt. Soc. Am. B 34, D46-D55 (2017).

22. K. Kneipp, Y. Wang, H. Kneipp, L. T. Perelman, I. Itzkan, R. R. Dasari, and M. S. Feld, "Single molecule detection using surface-enhanced Raman scattering (SERS)," Phys. Rev. Lett. 78, 1667-1670 (1997).

23. M. T. Hill and M. C. Gather, "Advances in small lasers," Nat. Photonics 8, 908-918 (2014).

24. R. Chikkaraddy, B. de Nijs, F. Benz, S. J. Barrow, O. A. Scherman, E. Rosta, A. Demetriadou, P. Fox, O. Hess, and J. J. Baumberg, "Singlemolecule strong coupling at room temperature in plasmonic nanocavities," Nature 535, 127-130 (2016).

25. S. Franke, M. Richter, J. Ren, A. Knorr, and S. Hughes, "Quantized quasinormal-mode description of nonlinear cavity-QED effects from coupled resonators with a Fano-like resonance," Phys. Rev. Res. 2, 033456 (2020).

26. F. Peyskens, A. Dhakal, P. V. Dorpe, N. L. Thomas, and R. Baets, "Surface enhanced Raman spectroscopy using a single mode nanophotonic plasmonic platform," ACS Photon. 3, 102-108 (2016).

27. J. Losada, A. Raza, S. Clemmen, A. Serrano, A. Griol, R. Baets, and A. Martínez, "SERS detection via individual bowtie nanoantennas integrated in $\mathrm{Si}_{3} \mathrm{~N}_{4}$ waveguides," IEEE J. Sel. Top. Quantum Electron. 25, 4600806 (2019).

28. Y. Bian and Q. Gong, "Bow-tie hybrid plasmonic waveguides," J. Lightwave Technol. 32, 4504-4509 (2014).

29. W.-C. Yue, P.-J. Yao, L.-X. Xu, and H. Ming, "All-dielectric bowtie waveguide with deep subwavelength mode confinement," Front. Phys. 13, 134207 (2018).

30. I. A. Pita, M. Kumbham, M. Schmidt, M. Gleeson, K. M. Ryan, C. Silien, and N. Liu, "Surface plasmon propagation enhancement via bowtie antenna incorporation in Au-mica block waveguides," Appl. Opt. 57, E50-E56 (2018).

31. A. Astorino, J. Lægsgaard, and K. Rottwitt, "The bowtie effect in cylindrical waveguides," J. Lightwave Technol. 36, 3309-3317 (2018).

32. N. A. Hatab, C.-H. Hsueh, A. L. Gaddis, S. T. Retterer, J.-H. Li, G. Eres, Z. Zhang, and B. Gu, "Free-standing optical gold bowtie nanoantenna with variable gap size for enhanced Raman spectroscopy," Nano Lett. 10, 4952-4955 (2010).

33. W. Zhu and K. B. Crozier, "Quantum mechanical limit to plasmonic enhancement as observed by surface-enhanced Raman scattering," Nat. Commun. 5, 5228 (2014).

34. H. Duan, A. I. Fernández-Domínguez, M. Bosman, S. A. Maier, and J. K. W. Yang, "Nanoplasmonics: classical down to the nanometer scale," Nano Lett. 12, 1683-1689 (2012).

35. G. Lévêque and O. J. F. Martin, "Optical interactions in a plasmonic particle coupled to a metallic film," Opt. Express 14, 9971-9981 (2006).

36. C. Ciracì, R. T. Hill, J. J. Mock, Y. Urzhumov, A. I. FernándezDomínguez, S. A. Maier, J. B. Pendry, A. Chilkoti, and D. R. Smith, "Probing the ultimate limits of plasmonic enhancement," Science 337, 1072-1074 (2012).

37. J. J. Baumberg, J. Aizpurua, M. H. Mikkelsen, and D. R. Smith, "Extreme nanophotonics from ultrathin metallic gaps," Nat. Mater. 18, 668-678 (2019).

38. C. Carnegie, J. Griffiths, B. de Nijs, C. Readman, R. Chikkaraddy, W. M. Deacon, Y. Zhang, I. Szabó, E. Rosta, J. Aizpurua, and J. J. Baumberg, "Room-temperature optical picocavities below $1 \mathrm{~nm}^{3}$ accessing single-atom geometries," J. Phys. Chem. Lett. 9, 7146-7151 (2018).

39. M. Barbry, P. Koval, F. Marchesin, R. Esteban, A. G. Borisov, J. Aizpurua, and D. Sánchez-Portal, "Atomistic near-field nanoplasmonics: reaching atomic-scale resolution in nanooptics," Nano Lett. 15, 3410-3419 (2015).
40. Y. Zhang, M. W. McCutcheon, I. B. Burgess, and M. Lončar, "Ultrahigh-Q TE/TM dual-polarized photonic crystal nanocavities," Opt. Lett. 34, 2694-2696 (2009).

41. M. W. McCutcheon, P. B. Deotare, Y. Zhang, and M. Lončar, "High-Qtransverse-electric/transverse magnetic photonic crystal nanobeam cavities," Appl. Phys. Lett. 98, 111117 (2011).

42. S. G. Johnson, S. Fan, P. R. Villeneuve, J. D. Joannopoulos, and L. A. Kolodziejski, "Guided modes in photonic crystal slabs," Phys. Rev. B 60, 5751-5758 (1999).

43. Y. Pennec, B. D. Rouhani, E. H. E. Boudouti, C. Li, Y. E. Hassouani, J. O. Vasseur, N. Papanikolaou, S. Benchabane, V. Laude, and A. Martinez, "Simultaneous existence of phononic and photonic band gaps in periodic crystal slabs," Opt. Express 18, 1430114310 (2010).

44. S.-G. Lee and R. Magnusson, "Essential differences between TE and TM band gaps in periodic films at the first Bragg condition," Opt. Lett. 44, 4658-4661 (2019).

45. D. J. Wilson, K. Schneider, S. Hönl, M. Anderson, Y. Baumgartner, L. Czornomaz, T. J. Kippenberg, and P. Seidler, "Integrated gallium phosphide nonlinear photonics," Nat. Photonics 14, 57-62 (2020).

46. R. Stockill, M. Forsch, G. Beaudoin, K. Pantzas, I. Sagnes, R. Braive, and S. Gröblacher, "Gallium phosphide as a piezoelectric platform for quantum optomechanics," Phys. Rev. Lett. 123, 163602 (2019).

47. COMSOL Inc., "COMSOL Multiphysics 5.0," https://www.comsol.com.

48. E. D. Palik, Handbook of Optical Constants of Solids (Academic, 1998).

49. W. L. Barnes, S. A. R. Horsley, and W. L. Vos, "Classical antennas, quantum emitters, and densities of optical states," J. Opt. 22, 073501 (2020).

50. A. Xomalis, R. Chikkaraddy, E. Oksenberg, I. Shlesinger, J. Huang, E. C. Garnett, A. F. Koenderink, and J. J. Baumberg, "Controlling optically driven atomic migration using crystal-facet control in plasmonic nanocavities," ACS Nano 14, 10562-10568 (2020).

51. R. Zhang, Y. Zhang, Z. C. Dong, S. Jiang, C. Zhang, L. G. Chen, L. Zhang, Y. Liao, J. Aizpurua, Y. Luo, J. L. Yang, and J. G. Hou, "Chemical mapping of a single molecule by plasmon-enhanced Raman scattering," Nature 498, 82-86 (2013).

52. L. Zhao, K. L. Kelly, and G. C. Schatz, "The extinction spectra of silver nanoparticle arrays: influence of array structure on plasmon resonance wavelength and width," J. Phys. Chem. B 107, 7343-7350 (2003).

53. R. Waldron, "Perturbation theory of resonant cavities," Proc. IEE C 107, 272-274 (1960).

54. O. Klein, S. Donovan, M. Dressel, and G. Grüner, "Microwave cavity perturbation technique: Part I: principles," Int. J. Infrared Millim. Waves 14, 2423-2457 (1993).

55. F. Vollmer and S. Arnold, "Whispering-gallery-mode biosensing: labelfree detection down to single molecules," Nat. Methods 5, 591-596 (2008).

56. A. F. Koenderink, M. Kafesaki, B. C. Buchler, and V. Sandoghdar, "Controlling the resonance of a photonic crystal microcavity by a near-field probe," Phys. Rev. Lett. 95, 153904 (2005).

57. F. Ruesink, H. M. Doeleman, R. Hendrikx, A. F. Koenderink, and E. Verhagen, "Perturbing open cavities: anomalous resonance frequency shifts in a hybrid cavity-nanoantenna system," Phys. Rev. Lett. 115, 203904 (2015).

58. J. Yang, H. Giessen, and P. Lalanne, "Simple analytical expression for the peak-frequency shifts of plasmonic resonances for sensing," Nano Lett. 15, 3439-3444 (2015).

59. T. Weiss, M. Mesch, M. Schäferling, H. Giessen, W. Langbein, and E. Muljarov, "From dark to bright: first-order perturbation theory with analytical mode normalization for plasmonic nanoantenna arrays applied to refractive index sensing," Phys. Rev. Lett. 116, 237401 (2016).

60. K. G. Cognée, H. M. Doeleman, P. Lalanne, and A. F. Koenderink "Cooperative interactions between nano-antennas in a high- $Q$ cavity for unidirectional light sources," Light Sci. Appl. 8, 115 (2019).

61. H. M. Lai, P. T. Leung, K. Young, P. W. Barber, and S. C. Hill, "Timeindependent perturbation for leaking electromagnetic modes in open systems with application to resonances in microdroplets," Phys. Rev. A 41, 5187-5198 (1990). 
62. P. T. Kristensen, J. R. de Lasson, and N. Gregersen, "Calculation, normalization, and perturbation of quasinormal modes in coupled cavity-waveguide systems," Opt. Lett. 39, 6359-6362 (2014).

63. P. Lalanne, W. Yan, K. Vynck, C. Sauvan, and J.-P. Hugonin, "Light interaction with photonic and plasmonic resonances," Laser Photon. Rev. 12, 1700113 (2018).

64. F. J. Rodrguez-Fortuño, A. Espinosa-Soria, and A. Martnez, "Exploiting metamaterials, plasmonics and nanoantennas concepts in silicon photonics," J. Opt. 18, 123001 (2016).

65. M. Frimmer and A. F. Koenderink, "Superemitters in hybrid photonic systems: a simple lumping rule for the local density of optical states and its breakdown at the unitary limit," Phys. Rev. B 86, 235428 (2012).

66. B. Gurlek, V. Sandoghdar, and D. Martín-Cano, "Manipulation of quenching in nanoantenna-emitter systems enabled by external detuned cavities: a path to enhance strong-coupling," ACS Photon. 5, 456-461 (2018).

67. M. Barth, S. Schietinger, S. Fischer, J. Becker, N. Nüsse, T. Aichele, B. Löchel, C. Sönnichsen, and O. Benson, "Nanoassembled plasmonic-photonic hybrid cavity for tailored light-matter coupling," Nano Lett. 10, 891-895 (2010).

68. J. Ho, Y. H. Fu, Z. Dong, R. Paniagua-Dominguez, E. H. H. Koay, Y. F. Yu, V. Valuckas, A. I. Kuznetsov, and J. K. W. Yang, "Highly directive hybrid metal-dielectric yagi-uda nanoantennas," ACS Nano 12 , 8616-8624 (2018).

69. E. Rusak, I. Staude, M. Decker, J. Sautter, A. E. Miroshnichenko, D. A. Powell, D. N. Neshev, and Y. S. Kivshar, "Hybrid nanoantennas for directional emission enhancement," Appl. Phys. Lett. 105, 221109 (2014). 